\title{
CATÁLOGO DE LOS UNIÓNIDOS (MOLLUSCA, UNIONIDAE) DE LA PENÍNSULA IBÉRICA CONSERVADOS EN EL MUSEO NACIONAL DE CIENCIAS NATURALES (CSIC)
}

\author{
O. Soriano (*), M. Villena (*) y M. S. Alonso (*)
}

\begin{abstract}
RESUMEN
Desde el año 1987 se están recuperando las colecciones del Museo Nacional de Ciencias Naturales. Durante 1999, entre otros trabajos, se han catalogado los 1363 ejemplares de uniónidos de la península Ibérica, que procedentes de las colecciones de González Hidalgo, Paz y Membiela, Azpeitia, Álvarez, Cobos, etc., se conservan en el museo.

El progresivo deterioro que, en la segunda mitad del siglo XX, han tenido los hábitats de las especies de la familia que aquí tratamos, ha producido, su desaparición en gran parte de las localidades de las que se conserva representación en las colecciones del museo.

La rápida regresión sufrida por las especies ibéricas, nos pone de manifiesto la necesidad de proceder a la recuperación y a la conservación de los ecosistemas acuáticos con presencia de especies pertenecientes a esta familia, antes de que se produzca su total desaparición. Por todo ello, se hace necesario conocer la información que se recoge en colecciones públicas o privadas y que podría contribuir a conocer la distribución que, en tiempos pasados, tenían estas especies.
\end{abstract}

Palabras clave: Catálogo, Unionidae, almejas de agua dulce, península Ibérica, Museo Nacional de Ciencias Naturales.

\section{ABSTRACT \\ Catalogue of Unionids (Mollusca, Unionidae) from Iberian peninsula preserved in the Museo Nacional of Ciencias Naturales}

From the 1987, we are working in the recuration process of the collections from the Museo Nacional de Ciencias Naturales (MNCN). In 1999, among other achievements, 1357 specimens of unionids from the Iberian Peninsula have been catalogued; these specimens are from the collections of González Hidalgo, Paz y Membiela, Azpeitia, Álvarez, Cobos, etc.

The habitats of the species of unionids experienced a gradual deterioration during the second half of the XXth century due to their relative intolerance to pollution. Actually, most collecting localities of the specimens in the MNCN are not suitable habitat for unionids today.

The fast regression suffered by the Iberian species underscores the need to proceed to the recovery and the conservation of the aquatic ecosystems where species belonging to this family are present, before their total disappearance takes place.

It is necessary to gather the information deposited in public and private collections, that could contribute to determine more accurately the past distribution of these species.

Keywords: Catalogue, Unionidae, freshwater mussels, Iberian peninsula, National Museum of Natural Sciences.

\footnotetext{
* Museo Nacional de Ciencias Naturales (C.S.I.C.). C/ José Gutiérrez Abascal nº 2, 28006 - Madrid
} 


\section{Introducción}

Desde el año 1987 se están recuperando y catalogando las colecciones del Museo Nacional de Ciencias Naturales debido al abandono que sufrieron desde el comienzo de la Guerra Civil Española. Durante 1999, entre otros trabajos, se han catalogado las colecciones de la Península Ibérica de la familia Unionidae, en las que se conserva material de autores como González Hidalgo, Paz y Membiela, Azpeitia, Álvarez, etc. Los cuales hicieron sus recolecciones desde mediados del siglo XIX hasta mediados del XX

Los ecosistemas acuáticos son de los más frágiles ecosistemas del mundo pero aún seguimos manipulándolos para cubrir nuestras necesidades a pesar de desconocer las consecuencias de nuestras actuaciones a largo plazo (Kaufman, 1992). Los factores que afectan a la extinción de los uniónidos son diversos y normalmente están interrelacionados, el progresivo deterioro del hábitat (cambios en la sedimentación, contaminación orgánica, con la consecuente disminución del oxígeno disuelto, lavado de campos agrícolas con aporte de pesticidas, acidificación y adición de metales pesados por explotaciones mineras, etc.) la desaparición de los peces hospedadores de los gloquidios, la explotación comercial de algunas especies y la introducción de especies exóticas han sido procesos frecuentes, sobre todo en la segunda mitad del siglo XX que ha llevado a la regresión de las poblaciones de las distintas especies de uniónidos (Bogan, 1993)

Este fenómeno se muestra muy patente al estudiar las colecciones de los museos y comparar la distribución de las especies en la época en que se hicieron las recolecciones y la distribución actual. La rápida regresión sufrida por las especies ibéricas, nos pone de manifiesto la necesidad de proceder a la recuperación y la conservación de ríos y tramos de ríos que contaban con la presencia de especies de la familia Unionidae antes de que se produzca la definitiva desaparición de las especies de esta familia en la Península Ibérica.

El presente trabajo aporta información inédita sobre la presencia de las distintas especies de uniónidos en diferentes localidades ibéricas.

\section{Material y métodos}

El material sujeto de nuestro trabajo, tanto el conservado en seco (conchas), la gran mayoría, como el conservado en alcohol, comenzó a ser recuperado básicamente (limpieza, reordenación, y reubicación en armarios especialmente acondicionados) durante el año 1989. Hasta 1999 los lotes de la colección no habían sido revisados nomenclaturalmente desde su primera determinación, lo cual abordamos en este trabajo.

La nomenclatura que usamos es la misma usada en la revisión sistemática de las náyades de Europa realizada por Nagel et al. (2001). Las sinonimias que listamos al principio de cada especie corresponden a los nombres no válidos, según Haas (1969), y con los que han sido nominadas cada una de las especies en la colección del museo. En el caso del género Anodonta Lamarck, 1799, la confirmación de la existencia de dos especies en la Europa mediterránea (Nagel et al., 1996), puede suponer, que al igual que en Italia en la península Ibérica se puedan encontrar las dos especies, pero como la diferenciación en función de la concha no es muy clara, asignamos la totalidad de los lotes a la especie $A$. cygnea (Linnaeus, 1758) hasta que posteriores estudios taxonómicos nos resuelvan este problema.

Para completar la información extrínseca de cada lote (localidad geográfica, recolector, fecha de captura, colección de procedencia, etc.) ha sido necesaria la recuperación de toda la información histórica recogida en catálogos, obras publicada por los autores de las colecciones y cualquier otra documentación que nos pudiera aportar datos sobre los lotes o ejemplares, aún así, en muchos casos no se conocen algunos de estos campos y por ello no son reflejados.

Una vez terminado el proceso de recopilación de la información, a cada lote (ejemplares de la misma especie, procedentes de una misma localidad y recolectados en una misma fecha) se le asignó el número de catálogo que corresponde a las colecciones de invertebrados no insectos del Museo Nacional de Ciencias Naturales y que se compone del acrónimo del Museo (MNCN), de un primer número referido al filum que se separa por un punto, de un segundo número correspondiente a la clase y que a su vez se separa por una barra de un tercer número que caracteriza al lote.

En el catálogo que ofrecemos, recogemos la especie, las sinonimias con que ha aparecido en las colecciones, el número de catálogo, la localidad de captura, la fecha de captura (siempre que se ha dispuesto de esta información), la colección de procedencia (se recoge el autor de la colección y su número de catálogo, siempre que el autor tuviera el lote catalogado. P. e.- Azpeitia siempre da un número de catálogo igual a todos los lotes de la misma 
especie), el recolector (siempre que se conozca), el modo de conservación (material en seco o en etanol $70^{\circ}$ ) y el número de ejemplares. Además, en aquellos lotes que se considere necesario se añaden observaciones en las que se especifica, por ejemplo, si el ejemplar ha sido estudiado o figurado en alguna publicación. A fin de ahorrar espacio y como la mayoría de los lotes son de procedencia española, sólo se indica el país de proceddencia cuando el país es Portugal.

Al final del catálogo de cada especie las figuras correspondientes (Fig. 1-6) muestran los mapas con la distribución en función del material conservado en el Museo Nacional de Ciencias Naturales de Madrid.

\section{Relación sistemática de los taxones}

Las colecciones del Museo Nacional de Ciencias Naturales conservan un total de 1363 ejemplares de uniónidos ibéricos, agrupados en 365 lotes de 5 especies. A continuación se ofrece un listado con la totalidad de los lotes ordenados taxonómicamente.

\section{Familia UNIONIDAE Fleming, 1828}

\section{Subfamilia Unioninae}

Género Unio Retzius, 1788

Unio pictorum (Linnaeus, 1758)

Unio pictorum pictorum (Linnaeus, 1758)

Mya pictorum Linnaeus, 1758. Syst. Nat., Ed. 10, 1: 671

Unio brindosianus Folin y Bérillon, 1874 (U. Moreleti var. brindosiana). Unio callipygus Drouët, 1893.

Unio cameratus Drouët, 1893.

Unio decurtatus Drouët, 1893.

Unio gravatus Drouët, 1893.

Unio hispanus Rossmässler, 1844.

Unio hispanus var. sphenoides Westerlund, 1892.

Unio limosellus Drouët, 1893.

Unio microdactylus Fagot in Locard, 1889.

Unio mucidus Morelet, 1845.

Unio sevillensis Kobelt, 1887.

Unio sphenoides Westerlund, 1892

MNCN 15.07/189: Río Miño, Tuy; Pontevedra; Colección Azpeitia; Macho; SECO; 1 ej.

Obs.: En la etiqueta antigua aparece como Unio bigerrensis Millet de la Turtaudière, 1843. Material estudiado en Azpeitia (1933: 210-212).

MNCN 15.07/226: Río Guadiana, Ciudad Real; Ciudad Real; Colección Azpeitia 1146; SECO; 3 ej.

Obs.: En la etiqueta antigua aparece como Unio callipygus Drouët, 1893. Material estudiado y figurado en Azpeitia (1933: 243-245, lám IV, fig. 21).
MNCN 15.07/238: Río Guadarrama, puente carretera MadridEl Escorial; Madrid; Colección Azpeitia 1059; Azpeitia; SECO; 17 ej.

Obs.: Los ejemplares se encontraban separados en tres lotes. En la etiqueta antigua aparece como Unio hispanus Moquin-Tandon in Rössmassler, 1844. Material estudiado en Azpeitia (1933: 316-320).

MNCN 15.07/240: Río Guadalquivir?, Sevilla?; Colección Azpeitia 1059; SECO; 4 ej.

Obs.: Azpeitia explica que los ejemplares son de Sevilla, seguramente del río Guadalquivir. El lote es de cuatro ejemplares; colectados por Hidalgo y Calderón, pero se encontraban separados en dos lotes. En la etiqueta antigua aparece como Unio hispanus Moquin-Tandon in Rössmassler, 1844. Material estudiado en Azpeitia (1933: 316-320).

MNCN 15.07/242: Río Guadalhorce; Málaga; Colección Azpeitia 1144; SECO; 1 ej.

Obs.: En la etiqueta antigua aparece como Unio sphenoides Westerlund. Material estudiado en Azpeitia (1933: 320-321).

MNCN 15.07/244: Río Tajo, Toledo; Toledo; Colección Azpeitia 1151; SECO; 4 ej.

Obs.: Azpeitia explica que las conchas que posee son bastante mayores que las de la descripción de Kobelt y las asocia a una "forma anormal". En la etiqueta antigua aparece como Unio sevillensis Kobelt, 1887. Material estudiado en Azpeitia (1933: 321-323).

MNCN 15.07/246: Río Tajo, Toledo; Toledo; Colección Azpeitia 1059; SECO; 1 ej.

Obs.: Según la publicación el colector es Pérez Arcas. En la etiqueta antigua aparece como Unio hispanus Moquin-Tandon in Rössmassler, 1844. Material estudiado en Azpeitia (1933: 316-320).

MNCN 15.07/270: Río Guadiana; Ciudad Real; Colección Azpeitia 1149; SECO; 1 ej.

Obs.: En la etiqueta antigua aparece como Unio decurtatus Drouët 1893.

MNCN 15.07/290: La Rivera de Huelva; Huelva; Colección Azpeitia 1059; Gavala; SECO; 2 ej.

Obs.. En la etiqueta antigua aparece como Unio hispanus Moquin-Tandon in Rossmässler, 1844. Material estudiado en Azpeitia (1933: 316-320).

MNCN 15.07/299: Arroyo Gavino, entre Alcolea del Río y Villaverde; Sevilla; Colección Azpeitia 1059; Gavala; SECO; 2 ej.

Obs.: Los ejemplares se encontraban separados en dos lotes. En la etiqueta antigua aparece como Unio hispanus Moquin-Tandon in Rossmässler, 1844. Material estudiado en Azpeitia (1933: 316-320).

MNCN 15.07/301: Río Jerte, Plasencia; Cáceres; Colección Azpeitia 1059; Hidalgo; SECO; 5 ej.

Obs.: Los ejemplares se encontraban separados en tres lotes. En la etiqueta antigua aparece como Unio hispanus Moquin-Tandon in Rossmässler, 1844. Material estudiado en Azpeitia (1933: 316-320).

MNCN 15.07/313: Río Mondego; Coimbra; Portugal; Colección Azpeitia 1040; Paz; SECO; 9 ej.

Obs.: En algunos de los ejemplares en la etiqueta pone Paz. En la etiqueta antigua aparece como Unio mucidus Morelet, 1845 .

MNCN 15.07/314: Río Duero, Soria; Soria; Colección Azpeitia 1040; Azpeitia; SECO; 5 ej. 
Obs.: En la etiqueta antigua aparece como Unio mucidus Morelet, 1845. Material estudiado en Azpeitia (1933: 344).

MNCN 15.07/317: Río Jarama, a 15 kms de Madrid; Madrid; Colección Azpeitia 1150; SECO; 2 ej.

Obs.. En la etiqueta antigua aparece como Unio limosellus Drouët, 1893.

MNCN 15.07/318: Portugal; Colección Azpeitia 1040; SECO; 3 ej.

Obs.: En la etiqueta antigua aparece como Unio mucidus Morelet, 1845.

MNCN 15.07/324: Río Guadiana, Villanueva de la Serena; Badajoz; Colección Azpeitia 1573; SECO; 1 ej.

Obs.: En la etiqueta antigua aparece como Unio microdactylus Fagot in Locard, 1889. Material estudiado y figurado en Azpeitia (1933: 273-276, lám. IX, fig. 50).

MNCN 15.07/328: Río Manzanares, Puerta de Hierro, Madrid; Madrid; Colección Azpeitia 1150; SECO; 1 ej.

Obs.: En la etiqueta antigua aparece como Unio limosellus Drouët, 1893.

MNCN 15.07/340: Arroyo Alberite, cortijo Alperchite, Arcos de la Frontera; Cádiz; Colección Azpeitia 1150; Gavala; SECO; 2 ej.

Obs.: Lote con dos etiquetas antiguas, en ellas aparece como Unio limosellus Drouët, 1893.

MNCN 15.07/389: Río Duero, Soria; Soria; Colección Azpeitia 1151; SECO; 5 ej.

Obs.: En la etiqueta antigua aparece como Unio sevillensis Kobelt. Material estudiado y figurado en Azpeitia (1933: p. 321-323, lám. VII, fig. 40 y 41).

MNCN 15.07/401: Río Miño, Tuy; Pontevedra; Colección Azpeitia 1040; SECO; 5 ej.

Obs.: Lote con dos etiquetas antiguas, una de ellas en francés y seguramente determinados por Hidalgo, en ellas aparece como Unio mucidus Morelet, 1845.

MNCN 15.07/471: Río Guadaira; Sevilla; Colección Azpeitia 1059; SECO; 8 ej.

Obs.: Los ejemplares se encontraban separados en tres lotes. 6 de ellos son ejemplares juveniles. En la etiqueta antigua aparecen como Unio hispanus Moquin-Tandon in Rossmässler, 1844. Material estudiado en Azpeitia (1933: 316-320).

MNCN 15.07/481: Tarragona; Tarragona; Colección Azpeitia 1059; Parellada e Hidalgo; SECO; 1 ej.

Obs.: En la etiqueta antigua pone Parellada, Tarragona; en la publicación cita a Parellada e Hidalgo como recolectores. En la etiqueta antigua aparece como Unio hispanus Moquin-Tandon in Rossmässler, 1844. Material estudiado en Azpeitia (1933: 316-320).

MNCN 15.07/487: Río Valdeazogues; Ciudad Real; Colección Azpeitia 1149; SECO; 1 ej.

Obs.: En la etiqueta antigua aparece como Unio decurtatus Drouët, 1893.

MNCN 15.07/506: Río Genil; Granada; Colección Paz y Membiela; Paz; SECO; 1 ej.

Obs.: Ejemplar separado del lote MNCN 15.07/1450 porque lleva escrita otra localidad en la concha. En la etiqueta antigua aparece como Unio moquinianus ? Dupuy, 1843.

MNCN 15.07/509: España; Colección Azpeitia 1049; SECO; 3 ej. Obs.: En la etiqueta antigua aparece como Unio brindosianus De Folin y Berillon, 1874.
MNCN 15.07/510: Río Tajo, Toledo; Toledo; Colección Azpeitia 1059; Martínez; SECO; 1 ej.

Obs.: En la etiqueta antigua aparece como Unio hispanus Moquin-Tandon in Rossmässler, 1844. Material estudiado en Azpeitia (1933: 316-320).

MNCN 15.07/551: Río Manzanares, cerca de Puerta de Hierro, Madrid; Madrid; SECO; 5 ej.

Obs.: En la etiqueta antigua aparece como Unio hispanus Rössmassler

MNCN 15.07/552: Río Genil, Loja; Granada; Colección Azpeitia 1059; Hidalgo; SECO; 1 ej.

Obs.: En la etiqueta antigua aparece como Unio hispanus Moquin-Tandon in Rossmässler, 1844. Material estudiado en Azpeitia (1933: 316-320).

MNCN 15.07/556: Río Guadalhorce; Málaga; Colección Azpeitia 1059; Hidalgo; SECO; 2 ej.

Obs.: Los ejemplares se encontraban separados en dos lotes. En la etiqueta antigua aparece como Unio hispanus Moquin-Tandon in Rossmässler, 1844. Material estudiado y figurado en Azpeitia (1933: 316-320, lám. VII, fig. 38).

MNCN 15.07/563: Ribeira Lucefeci, Alentejo, Portugal; SECO; 3 ej.

Obs.: En la etiqueta antigua aparece como Unio littoralis Lamarck.

MNCN 15.07/569: Ribeira Lucefeci, Alentejo, Portugal; Colección Paz y Membiela; Paz; SECO; 2 ej.

Obs.: En la etiqueta antigua aparece como Unio mucidus Morelet, 1845.

MNCN 15.07/573: Río Zadorra; Álava; Colección Azpeitia 1149; SECO; 1 ej.

Obs.. En la etiqueta antigua aparece como Unio decurtatus Drouët, 1893.

MNCN 15.07/576: Río Cea; León; Colección Azpeitia 1149; SECO; 4 ej.

Obs.: En la etiqueta antigua aparece como Unio decurtatus Drouët.

MNCN 15.07/577: Río Duero; Zamora; Colección Azpeitia 1149; SECO; 2 ej.

Obs.: En la etiqueta antigua aparece como Unio decurtatus Drouët, 1893.

MNCN 15.07/581: España; Colección Azpeitia; SECO; 1 ej. Obs.: En la etiqueta antigua aparece como Unio limosellus Drouët, 1893.

MNCN 15.07/583: Río Tajo; SECO; 7 ej.

Obs.: Lote con una etiqueta antigua que indica que los ejemplares son del género Unio y procedentes del Tajo.

MNCN 15.07/769: Lagunas de Ruidera; Ciudad Real; Colección Azpeitia 1146; Boscá; SECO; 4 ej.

Obs.: En la etiqueta antigua aparece como Unio callipygus Drouët, 1893. Material estudiado en Azpeitia (1933: 243-245)

MNCN 15.07/773: Sevilla?; Colección Azpeitia 1144; SECO; 10 ej.

Obs.: Los ejemplares se encontraban separados en tres lotes. La localidad seguramente es Río Guadaira en concordancia con el libro de Azpeitia. En la etiqueta antigua aparece como Unio sphenoides Westerlund, 1892. Material estudiado en Azpeitia (1933: 320-321).

MNCN 15.07/785: Río Tajo, Aranjuez; Madrid; Colección Azpeitia 1152; SECO; 6 ej. 
Obs.: Los ejemplares se encontraban separados en dos lotes. En la etiqueta antigua aparece como Unio jourdheuili Ray in Locard, 1882.

MNCN 15.07/1450: Río Támega; Colección Paz y Membiela; Paz; SECO; 1 ej.

Obs.: El lote tiene dos etiquetas antiguas y era de dos ejemplares, pero uno de ellos (MNCN 15.07/506) llevaba escrita otra localidad en la concha. En la etiqueta antigua aparece como Unio moquinianus ? Dupuy, 1843.

MNCN 15.07/1451: Río Tajo, Lisboa; Portugal. SECO; 2 ej. Obs.: En la etiqueta antigua pone Paz. Material seguramente procedente de la colección Paz y Membiela y aparece como Unio baeticus Kobelt, 1887.

MNCN 15.07/1453: Río Guadaira, cerca de Sevilla; Sevilla; Calderón; SECO; 2 ej.

Obs.: En la etiqueta antigua aparece como Unio baeticus Kobelt, 1887.

MNCN 15.07/1456: Río Zigl ?; Salamanca; Cisterna; SECO; 5 ej. Obs.: Lote con dos etiquetas antiguas, una de ellas del laboratorio de malacología en ambas aparece como Unio mucidus Morelet, 1845.

MNCN 15.07/1458: Extremadura; SECO; 3 ej.

Obs.: Lote con una etiqueta antigua procedente del laboratorio de malacología en ella aparece como Unio pictorum Linnaeus, 1758.

MNCN 15.07/1459: Río Miño, Tuy; Pontevedra; JUL/1879; SECO; 1 ej.

Obs.: Lote con dos etiquetas antiguas, en ellas aparece como Unio cameratus Drouët, 1893.

MNCN 15.07/1462: Río Guadiana; Ciudad Real; SECO; 5 ej. Obs.: En la etiqueta antigua aparece como Unio dactylus Morelet, 1845.

MNCN 15.07/1473: Río Tajo, Badajoz; Colección Azpeitia 1157; SECO; 6 ej.

Obs.: En la etiqueta antigua aparece como Unio gravatus v.? Drouët, 1893.

MNCN 15.07/1474: Río Miño, Tuy; Pontevedra; Colección Azpeitia 1040; SECO; 1 ej.

Obs.: En la etiqueta antigua aparece como Unio mucidus Morelet, 1845.

MNCN 15.07/1475: Lagunas de Ruidera; Ciudad Real; Colección Azpeitia 1157; SECO; 1 ej.

Obs.: En la etiqueta antigua aparece como Unio gravatus Drouët, 1893.

MNCN 15.07/1476: Lagunas de Ruidera; Ciudad Real; Colección Azpeitia 1039; Boscá; SECO; 1 ej.

Obs.: En la etiqueta antigua aparece como Unio dactylus Morelet, 1845.

MNCN 15.07/1484: Río Duero, Soria; Soria; Colección Hidalgo; SECO; 6 ej.

MNCN 15.07/1536: Río Tajo, Villarrubia de Santiago; Toledo; Colección Julio Álvarez; 1959?; SECO; 19 ej.

MNCN 15.07/1539: España ?; SECO; 8 ej.

Obs.: Material asociado con dudas como procedente de España. En la etiqueta antigua aparece como Unio littoralis?

MNCN 15.07/1545: Río Guadaira; Sevilla; Colección Azpeitia 1144; SECO; 2 ej.

Obs.: En la etiqueta antigua aparece como Unio sphenoides Westerlund, 1892. Material estudiado en Azpeitia (1933: 320-321).
MNCN 15.07/1546: Río Tajo, Badajoz; Badajoz; Colección Azpeitia 1144; SECO; 5 ej.

Obs.: Los ejemplares se encontraban separados en dos lotes. En la etiqueta antigua aparece como Unio sphenoides Westerlund, 1892; Azpeitia (1933: 320-321).

MNCN 15.07/1548: Río Lima; SECO; 2 ej.

Obs.: En la etiqueta antigua aparece como Unio mucidus Morelet, 1845.

MNCN 15.07/1559: Río Manzanares, Hoyo de Manzanares; Madrid; Colección Julio Álvarez; 3/OCT/1960; Fernández y Nieto; SECO; 7 ej.

Obs.: En la etiqueta antigua aparece como Unio microdactylus Fagot in Locard, 1889.

MNCN 15.07/1573: Río Guadaira; Sevilla; Colección Azpeitia 1059; Calderón; SECO; 2 ej.

Obs.: Los ejemplares se encontraban separados en dos lotes. En la etiqueta antigua aparece como Unio hispanus Moquin-Tandon in Rossmässler, 1844. Material estudiado en Azpeitia (1933: 316-320.

MNCN 15.07/1632: Portugal; SECO; 1 ej.

Obs.: En la etiqueta antigua pone Unio mucidus Morelet, Portugal, env. Bocage?.

MNCN 15.07/1639: Río Guadiana, Ciudad Real; Ciudad Real; SECO; 2 ej

Obs.: Lote con una etiqueta antigua pone sólo la localidad.

MNCN 15.07/1644: Río Tajo, Presa de Valdajas, Villarrubia de Santiago; Toledo; Colección Julio Álvarez; 11/ABR/1964; R. Alvarado; SECO; 1 ej.

Obs.: En la etiqueta antigua aparece como Unio sphenoides Westerlund, 1892.

MNCN 15.07/1657: Madrid; SECO; 1 ej.

MNCN 15.07/1661: Río Duero, Soria; Soria; Colección Azpeitia 1150; SECO; 5 ej.

Obs.: En la etiqueta antigua aparece como Unio limosellus Drouët, 1893. Material estudiado en Azpeitia (1933: 384).

MNCN 15.07/1662: Aluviones del río Jarama, San Martín de la Vega; Madrid; Colección Azpeitia 1150; SECO; 5 ej. Obs.: Es conveniente leer la etiqueta antigua de gran tamaño en la que Azpeitia realiza apreciaciones de los ejemplares y otorga a uno de ellos, estudiado por Drouët, la categoría de "casi" tipo. Esta forma de anotación es común en Azpeitia, ya que cuando observaba mucha similitud entre su ejemplar y el tipo figurado lo denominaba así o, simplemente "tipo". En la etiqueta antigua aparece como Unio limosellus Drouët, 1893.

MNCN 15.07/1666: Lagunas de Ruidera; Ciudad Real; 31/MAY-3/JUN/1914; Boscá; SECO; 12 ej.

Obs.: Lote con tres etiquetas antiguas, en ellas aparece como Unio callipygus Drouët, 1893.

MNCN 15.07/1687: Lagunas de Ruidera; Ciudad Real; Boscá; SECO; 1 ej.

Obs.: En la etiqueta antigua aparece como Unio gravatus Drouët, 1893.

MNCN 15.07/1710: Lozoya del Valle, término municipal de Pinilleja; Madrid; L. Fernández Aguilar; SECO; 11 ej.

MNCN 15.07/1723: Río Guadiana, Ciudad Real; Ciudad Real; SECO; 1 ej.

Obs.: En la etiqueta antigua aparece como Unio callipygus Drouët, 1893.

MNCN 15.07/1735: Toledo; Toledo; SECO; 1 ej. Obs.: El ejemplar tiene una etiqueta pegada. 
MNCN 15.07/1776: Río Manzanares, Hoyo de Manzanares; Madrid; Colección Julio Álvarez; 3/OCT/1960; Fernández y Nieto; SECO; 13 ej.

Obs.: En la etiqueta antigua aparece como Unio microdactylus Fagot in Locard, 1889.

MNCN 15.07/1778: Río Jándula; Jaén; Colección Julio Álvarez; 16/OCT/1961; SECO; 15 ej.

Obs.: En la etiqueta antigua aparece como Unio sphenoides Westerlund, 1892.

MNCN 15.07/1779: Río Tajo, presa de San Julián; Toledo; Colección Álvarez; 2/AGO/1962; J. Álvarez; SECO; 10 ej.

MNCN 15.07/1795: Río Jarama, puente de Arganda, Arganda; Madrid; Colección Julio Álvarez; 24/ABR/1961; SECO 2 ej.

Obs.: En la etiqueta antigua aparece como Unio limosellus Drouët, 1893.

MNCN 15.07/1796: Río Tajo, Villarrubia de Santiago; Toledo; Colección Julio Álvarez; 24/MAR/1962; SECO; 2 ej. Obs.: En la etiqueta antigua aparece como Unio decurtatus Drouët, 1893.

MNCN 15.07/1797: Río Miño, Tuy; Pontevedra; Colección Julio Álvarez; SECO; 1 ej.

Obs.: En la etiqueta antigua aparece como Unio decurtatus Drouët, 1893

MNCN 15.07/1799: Río Jándula; Jaén; Colección Julio Álvarez; 16/OCT/1961; M. Nieto; SECO; 1 ej.

Obs.. En la etiqueta antigua aparece como Unio sphenoides Westerlund.

MNCN 15.07/1802: Río San Buriel, Cerceda; Madrid; 21/MAY/1960; M. Nieto; SECO; 1 ej.

Obs.: En la etiqueta antigua pone río Manzanares, pero el río que pasa por Cerceda es el San Buriel tributario del Manzanares y aparece como Unio consentaneus Rössmassler.

MNCN 15.07/1806: Don Benito; Badajoz; SECO; 12 ej.

MNCN 15.07/1860: Río Mondego, Coimbra; Portugal; Colección Azpeitia 1157; SECO; 1 ej.

Obs.: En la etiqueta antigua aparece como Unio gravatus Drouët, 1893.

MNCN 15.07/3279: Río Ubieza, Piña de Campos; Palencia; Colección Ortiz de Zárate; AGO/1954; Altimira; SECO 1 ej.

MNCN 15.07/3280: Río Ubieza, Piña de Campos; Palencia; Colección Ortiz de Zárate; AGO/1954; Altimira; SECO; 1 ej.

MNCN 15.07/3667: Canal Imperial de Aragón; Zaragoza; IFIE (Instituto Forestal de Investigaciones y experimentación); III/1946; SECO; 1 ej.

MNCN 15.07/5118: Río Ulla; Azpeitia 1049; Cisneros; SECO; 4 ej.

Obs.: Según anotaciones de Azpeitia son los ejemplares juveniles de $U$. cameratus descritos por Drouët En la etiqueta antigua aparece como Unio brindosianus De Follin y Berillon, 1874. Material estudiado y figurado en Azpeitia (1933: 420-422, lám. 10, fig 57).

MNCN 15.07/5214: Río Tajo, Villarrubia de Santiago; Toledo; Colección Álvarez; 24/MAR/1961; Julio Álvarez; SECO; 10 ej.

Obs.: En la etiqueta antigua aparece como Unio sevillensis Kobelt, 1887.
MNCN 15.07/5216: Río Tiétar, Buenaventura; Toledo; SECO; 4 ej.

MNCN 15.07/5218: Río Guadamez, Vega de los Maderos, Don Benito; Badajoz; Verano/1941; SECO; 9 ej.

MNCN 15.07/5219: Río Guadiana, Don Benito; Badajoz; SECO; 1 ej

MNCN 15.07/5220: Río Guadamez, Vega de los Maderos, Don Benito; Badajoz; Verano/1941; Sr. Bayón; SECO; 1 ej.

MNCN 15.07/5221: Río Guadamez, Vega de los Maderos, Don Benito; Badajoz; Verano/1941; SECO; 20 ej.

MNCN 15.07/5224: Río Alberche, Cazalegas; Toledo; SECO; 2 ej.

MNCN 15.07/5244: Pamplona; Navarra; SECO; 1 ej. Obs.: Material extraído de otro lote por pertenecer a una especie diferente.

MNCN 15.07/5251: Río Guadiana, Badajoz; Badajoz; Colección Cobos; J. Rutllant; SECO; 1 ej.

Obs.: En la etiqueta antigua aparece como Unio microdactylus Fagot in Locard.

MNCN 15.07/5253: Río Vivar, puente en carretera de Burguillo; Sevilla; Colección Cobos; MAY/1965; L. García; SECO; 6 ej.

Obs.. En la etiqueta antigua aparece como Unio $s p$.

MNCN 15.07/5257: Río Ebro, Cenicero; Logroño; Colección Ortiz de Zárate; SECO; 2 ej.

Obs.: En la etiqueta antigua aparece como Unio requieni Michaud.

MNCN 15.07/5258: Arroyo de la Mujer, San Roque; Cádiz; Colección Cobos; AGO/1965; L. Ramírez; SECO; 8 ej. Obs.: En la etiqueta antigua aparece como Unio sphenoides Westerlund.

MNCN 15.07/5259: Río Cea, Valdescorriel; Zamora; Colección Cobos; 30/AGO/1978; L. Romero; SECO; 6 ej.

MNCN 15.07/5262: Río Tajo, Talavera de la Reina; Toledo; IFIE (Instituto Forestal de Investigaciones y Experimentación); 8/NOV/1943; SECO; 5 ej.

MNCN 15.07/5263: Río Guadiana, Badajoz; Colección Cobos; OCT/1951; SECO; 2 ej.

MNCN 15.07/5264: Río Tajo, Toledo; IFIE (Instituto Forestal de Investigaciones y Experimentación); 15/JUL/1942; SECO; 8 ej.

Obs.: En la etiqueta antigua aparece como Unio $s p$.

MNCN 15.07/5268: Río Alberche, Escalona; Toledo; IFIE (Instituto Forestal de Investigaciones y Experimentación); 15/OCT/1945; SECO; 1 ej.

Obs.: En la etiqueta antigua aparece como Unio requieni.

MNCN 15.07/5270: Río Tajo, Talavera de la Reina; Toledo; IFIE (Instituto Forestal de Investigaciones y Experimentación); 6/NOV/1943; SECO; 3 ej.

Obs.: En la etiqueta antigua aparece como Unio requieni Michaud.

MNCN 15.07/5274: Río Tajo, Talavera de la Reina; Toledo; IFIE (Instituto Forestal de Investigaciones y Experimentación); SECO; 2 ej.

Obs.: En la etiqueta antigua aparece como Unio requieni Michaud.

MNCN 15.07/5275: Río Salor, Aliseda; Cáceres; IFIE (Instituto Forestal de Investigaciones y Experimentación); 23/JUL/1949; SECO

Obs.: En la etiqueta antigua aparece como Unio requieni Michaud.

MNCN 15.07/5276: Río Carrión, Palencia; Palencia; IFIE (Instituto Forestal de Investigaciones y Experimentación); 25/NOV/1943; SECO; 1 ej. 


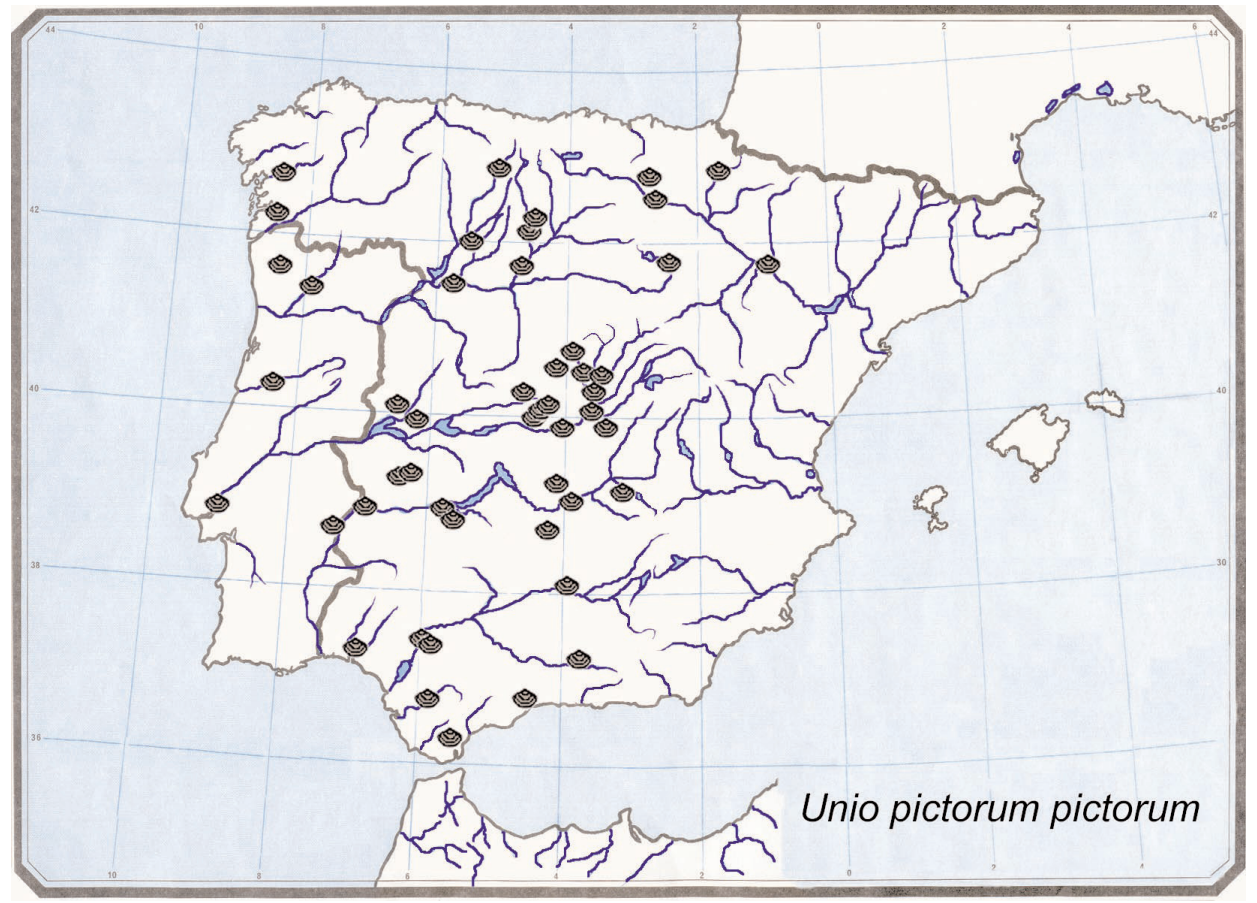

Fig. 1.- Distribución del material contenido en las Colecciones del MNCN de Unio pictorum pictorum (Linnaeus, 1757).

Fig. 1.- Distribution of the Unio pictorum pictorum (Linnaeus, 1757) material from the Spanish MNCN Collections.

Obs.: En la etiqueta antigua aparece como "Unio timidus Philipson".

MNCN 15.07/5277: Río Jarama, San Fernando de Henares; Madrid; IFIE (Instituto Forestal de Investigaciones y Experimentación); SECO; 26 ej.

Obs.: La etiqueta antigua lleva la fecha de captura que se lee muy mal y la especie aparece determinada como Unio requieni Michaud.

MNCN 15.07/5278: Río Pisuerga, Valladolid; IFIE (Instituto Forestal de Investigaciones y Experimentación); 23/JUN/1945; SECO; 15 ej.

Obs.: En la etiqueta antigua aparece como Unio requieni.

MNCN 15.07/5279: Río Jarama, "La Isla", San Fernando de Henares; Madrid; IFIE (Instituto Forestal de Investigaciones y Experimentación); 12/AGO/1942; SECO; 28 ej.

Obs.: En la etiqueta antigua aparece como Unio requieni.

MNCN 15.07/5280: Río Henares, Mejorada del Campo; Madrid; IFIE (Instituto Forestal de Investigaciones y Experimentación); 4/AGO/1943; SECO; 17 ej.

Obs.: En la etiqueta antigua aparece como Unio requieni.

MNCN 15.07/5282: Río Tajo, Talavera de la Reina; Toledo; IFIE (Instituto Forestal de Investigaciones y Experimentación); 6/NOV/1943; SECO; 4 ej.

MNCN 15.07/5283: Río Tiétar, Malpartida de Cáceres; Cáceres; IFIE (Instituto Forestal de Investigaciones y Experimentación); 7/MAY/1944; SECO; 4 ej.
Obs.: En la etiqueta antigua aparece como Unio $s p$.

MNCN 15.07/5284: Río Arrago, Moraleja; Cáceres; IFIE (Instituto Forestal de Investigaciones y Experimentación); SECO; 1 ej.

Obs.: En la etiqueta antigua aparece como Unio requieni.

MNCN 15.07/5292: Río Cea, Valdescorriel, Zamora; Colección Cobos; 30/AGO/1978; L. Romero; 3 ej.

Unio pictorum mancus Lamarck, 1819

Unio manca Lamarck, 1819, Hist. Nat. Anim. Sans vert., Part 6 1:80. Unio aleroni Companyo y Massot, 1845.

Unio almenarensis Drouët, 1893.

Unio courquinianus Bourguignat, 1865.

Unio elongatulus Pfeiffer, 1825.

Unio graellsianus Bourguignat, 1865

Unio jourdheuili Ray in Locard, 1882.

Unio moquinianus Dupuy, 1843.

Unio penchinatianus Bourguignat, 1865.

Unio penchinatianus var. minor Azpeitia, 1933.

Unio requieni Michaud, 1831.

Unio turtoni Payraudeau, 1826.

Unio valentinus Rossmässler, 1854.

MNCN 15.07/253: Valencia; Colección Hidalgo; SECO; 1 ej. Obs.: La etiqueta antigua no se corresponde con las de la colección Hidalgo y aparece como Unio courquinianus Bourguignat, 1864. 
MNCN 15.07/284: Canales del Turia; Valencia; Colección Azpeitia 1154; SECO; 1 ej.

Obs.: En la etiqueta antigua aparece como Unio graellsianus Bourguignat, 1864. Material estudiado en Azpeitia (1933: 273-275).

MNCN 15.07/322: Río Támega, Galicia; Colección Azpeitia 1156; SECO; 1 ej.

Obs.: En la etiqueta antigua aparece como Unio moquinianus Dupuy, 1843.

MNCN 15.07/346: Río Sió, Balaguer; Lérida; Colección Azpeitia 1572; SECO; 1 ej.

Obs.: En la etiqueta antigua aparece como Unio ovuliformis Locard, 1899. Material estudiado en Azpeitia (1933: 236-239).

MNCN 15.07/364: Río Ebro, Milagro; Navarra; Colección Azpeitia 1058; SECO; 2 ej.

Obs.: En la etiqueta antigua aparece como Unio turtoni Payraudeau, 1826.

MNCN 15.07/428: Albufera de Valencia; Valencia; Colección Azpeitia 1159; SECO; 9 ej.

Obs.: Se ha sacado del lote un ejemplar que pertenece al género Anodonta. En la etiqueta antigua aparece como Unio valentinus Rössmassler, 1854.

MNCN 15.07/430: Albufera de Valencia; Valencia; Colección Azpeitia 1159; SECO; 8 ej.

Obs.: En la etiqueta antigua aparece como Unio valentinus Rössmassler, 1854.

MNCN 15.07/447: Albufera de Valencia; Valencia; Colección Azpeitia 1058; Reyes; SECO; 1 ej.

Obs: En la etiqueta antigua aparece como Unio turtoni Payreaudeau, 1826

MNCN 15.07/453: Albufera de Valencia; Valencia; Boscá; SECO; 12 ej.

MNCN 15.07/454: Albufera de Valencia; Valencia; Boscá; SECO; 12 ej.

MNCN 15.07/492: Valencia; Colección Hidalgo; SECO; 5 ej. Obs.: Dos de los ejemplares son juveniles. En la etiqueta antigua aparece como Unio valentinus Rössmassler, 1854.

MNCN 15.07/549: Río Ebro, La Zaida; Zaragoza; Colección Azpeitia 1058; SECO; 13 ej.

Obs.: En la etiqueta antigua aparece como Unio turtoni Payreaudeau, 1826

MNCN 15.07/564: Dehesa al Norte del Paso, Albufera de Valencia; Valencia; Colección Hidalgo; SECO; 4 ej. Obs.: Material recolectado en canto rodado grueso entre 0 y 0.3 metros. En la etiqueta antigua aparece como Unio turtoni valentinus Rössmassler, 1854.

MNCN 15.07/571: Albufera de Valencia; Valencia; Colección Hidalgo; SECO; 3 ej.

Obs.: En la etiqueta antigua aparece como Unio turtoni valentinus Payreaudeau, 1826.

MNCN 15.07/757: Laguna de Almenara; Castellón; Colección Azpeitia 962; Boscá; SECO; 5 ej.

Obs.: Los ejemplares proceden de Boscá y son de la misma localidad que los que Drouët utilizó para la descripción de la especie (topotipos). En la etiqueta antigua aparecen como Unio almenarensis Drouët, 1893. Material estudiado en Azpeitia (1933: 276-277).

MNCN 15.07/775: Lago de Bañolas; Gerona; Colección Azpeitia 1050; SECO; 10 ej.
Obs.: En la etiqueta antigua aparecen como Unio penchinatianus Bourguignat, 1864. Material estudiado en Azpeitia (1933: 277-281, lám. 5, fig. 30).

MNCN 15.07/777: España; Colección Azpeitia 1160 (ex-Coll. Pérez-Arcas); SECO; 1 ej.

Obs.: Azpeitia indica que el ejemplar procede de la colección Pérez Arcas. En la etiqueta antigua aparece como Unio aleroni Companyo y Massot, 1845. Material estudiado en Azpeitia (1933: 261-265).

MNCN 15.07/779: España ?; Colección Azpeitia 1160; SECO; 4 ej.

Obs.: En la etiqueta antigua aparece como Unio aleroni Companyo y Massot, 1845.

MNCN 15.07/781: Río Júcar, Algemesí; Valencia; Colección Azpeitia 1154; SECO; 3 ej.

Obs.: En la etiqueta antigua aparece como Unio graellsianus Bourguignat, 1864. Material estudiado en Azpeitia (1933: 273-275, fig. 28b).

MNCN 15.07/783: Albufera de Valencia; Valencia; Colección Hidalgo; SECO; 2 ej.

Obs.: En la etiqueta antigua aparece como Unio courquinianus Bourguignat, 1864.

MNCN 15.07/823: Albufera de Valencia; Valencia; Colección Azpeitia 1170; SECO; 1 ej.

Obs.: En la etiqueta antigua aparece como Unio valentinus Rossmässler, 1854. Material estudiado y figurado en Azpeitia (1933: 266-268, lám. IV, fig. 26).

MNCN 15.07/1448: Río Oñar; Gerona; SECO; 2 ej.

Obs.: En la etiqueta antigua aparece como Unio hispanus Moquin-Tandon in Rossmässler, 1844.

MNCN 15.07/1454: Lago de Bañolas; Gerona; Colección J. Álvarez; 1865; SECO; 7 ej.

Obs.: En la etiqueta antigua aparece como Unio penchinatianus Bourguignat, 1864.

MNCN 15.07/1460: Riera de Osor, Anglés; Gerona; SECO; 3 ej. Obs.: En la etiqueta antigua pone que hay 4 ejemplares y aparece como Unio moquinianus Dupuy, 1843.

MNCN 15.07/1479: Extremadura; Paz; SECO; 1 ej. Obs.: En la etiqueta antigua aparece como Unio requieni Michaud, 1831.

MNCN 15.07/1537: Albufera de Valencia; Valencia; SECO; 1 ej.

MNCN 15.07/1550: Laguna de Almenara; Castellón; SECO; 5 ej.

Obs.: En la etiqueta antigua aparece como Unio valentinus Rossmässler, 1854.

MNCN 15.07/1566: Valencia; SECO; 3 ej.

Obs.: Alguno de los ejemplares lleva una etiqueta con el número 59 pegado. En la etiqueta antigua aparece como Unio valentinus Rossmässler, 1854.

MNCN 15.07/1567: Albufera de Valencia; Valencia; SECO; 5 ej. Obs.: En fango líquido entre 0.2 y 0.3 metros a unos 200 metros de la extremidad norte de la dehesa. En la etiqueta antigua aparece como Unio turtoni valentinus Rossmässler, 1854.

MNCN 15.07/1575: Río Tiétar; Toledo; Colección J. Álvarez; SECO; 1 ej.

Obs.: Los ejemplares se encontraban junto con el lote MNCN 15.07/1777 P. littoralis. En la etiqueta antigua aparece como Unio elongatulus Pfeiffer, 1825. 


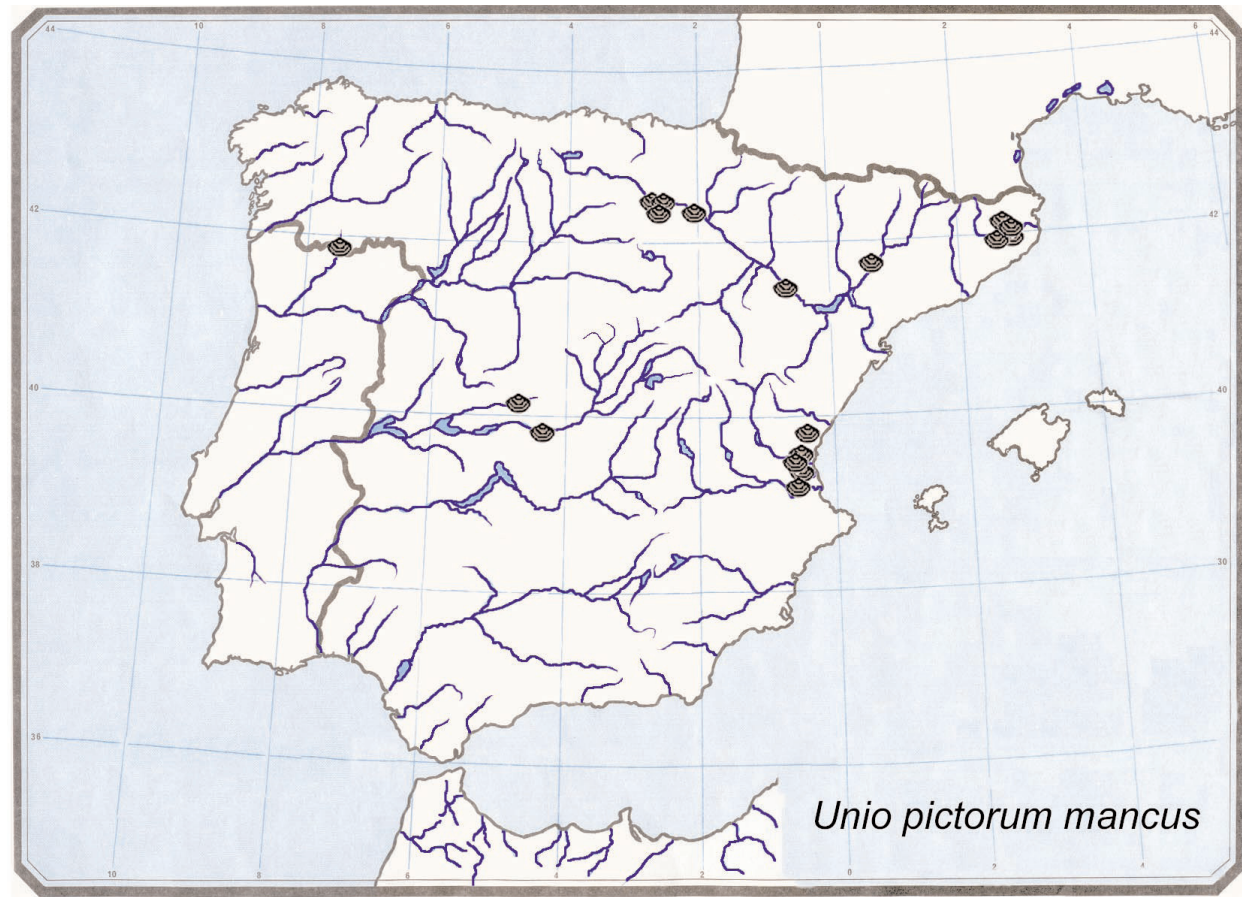

Fig. 2.- Distribución del material contenido en las Colecciones del MNCN de Unio pictorum mancus Lamarck, 1819.

Fig. 2.- Distribution of the Unio pictorum mancus Lamarck, 1819 material from the Spanish MNCN Collections.

MNCN 15.07/1590: Albufera de Valencia; Valencia; SECO; 1 ej Obs.: En la etiqueta antigua aparece como Unio aleroni Companyo y Massot, 1845.

MNCN 15.07/1592: Canal de Catarroja; Valencia; SECO; 1 ej. Obs.: En la etiqueta antigua aparece como Unio valentinus Rössmassler, 1854.

MNCN 15.07/1599: Albufera de Valencia; Valencia; SECO; 1 ej. Obs.: Se han sacado del lote dos ejemplares de $M$. margaritifera. En la etiqueta antigua aparece como Unio courquinianus Bourguignat, 1864.

MNCN 15.07/1621: Carrer del Saler, Albufera de Valencia; Valencia. SECO; 5 ej.

Obs.: Material recolectado cerca de la desembocadura, fango compacto de humus $0,5-0,75$ metros.

MNCN 15.07/1633: Albufera de Valencia; Valencia; SECO; 2 ej.

Obs.: Los ejemplares llevan marcado el número 22 en la concha. En la etiqueta antigua aparece como Unio courquinianus Bourguignat, 1864.

MNCN 15.07/1641: Dehesa al Norte del Paso, Albufera de Valencia; Valencia; SECO; 3 ej.

Obs.: Material recolectado en arena entre 0 y 0,5 metros. En la etiqueta antigua aparece como Unio turtoni valentinus Payraudeau.

MNCN 15.07/1658: Dehesa al Norte del Paso, Albufera de Valencia; Valencia; SECO; 1 ej.

Obs.: En la etiqueta antigua aparece como Unio turtoni Payreaudeau, 1826.
MNCN 15.07/1667: Albufera de Valencia; Extremidad Norte de la Dehesa, cerca de la salida de Carrer del Saler; Valencia; SECO; 3 ej.

Obs.: Material recolectado en fango arenoso entre 0,3 y 0,5 metros. En la etiqueta antigua aparece como Unio turtoni valentinus Payraudeau.

MNCN 15.07/1734: Valencia; SECO; 2 ej.

Obs.: En la etiqueta antigua parece que pone Acequia de Almenara y aparece como Unio aleroni Companyo y Massot, 1845.

MNCN 15.07/1742: Albufera de Valencia, Dehesa entre la Mata del Fanch y Mateta de la Sequieta; Valencia; SECO; 2 ej.

Obs.: Material recolectado en arena entre 0 y 0,5 metros. En la etiqueta antigua aparece como Unio turtoni valentinus Payraudeau.

MNCN 15.07/1744: Albufera de Valencia; Valencia; 1853; SECO; 9 ej

Obs.: En la etiqueta antigua aparece como Unio valentinus Rossmässler, 1854.

MNCN 15.07/1798: Lago de Bañolas; Gerona; Colección J. Álvarez; SECO; 2 ej.

Obs.: En la etiqueta antigua aparece como Unio penchinatianus Bourguignat, 1864.

MNCN 15.07/1804: Río Oñar; Gerona; SECO; 1 ej.

MNCN 15.07/3267: Río Najerilla, Nájera; Logroño; Colección Ortiz de Zárate $\mathrm{n}^{0} 3$; 15/SEP/1955; M. Calatayud; SECO; 1 ej. 


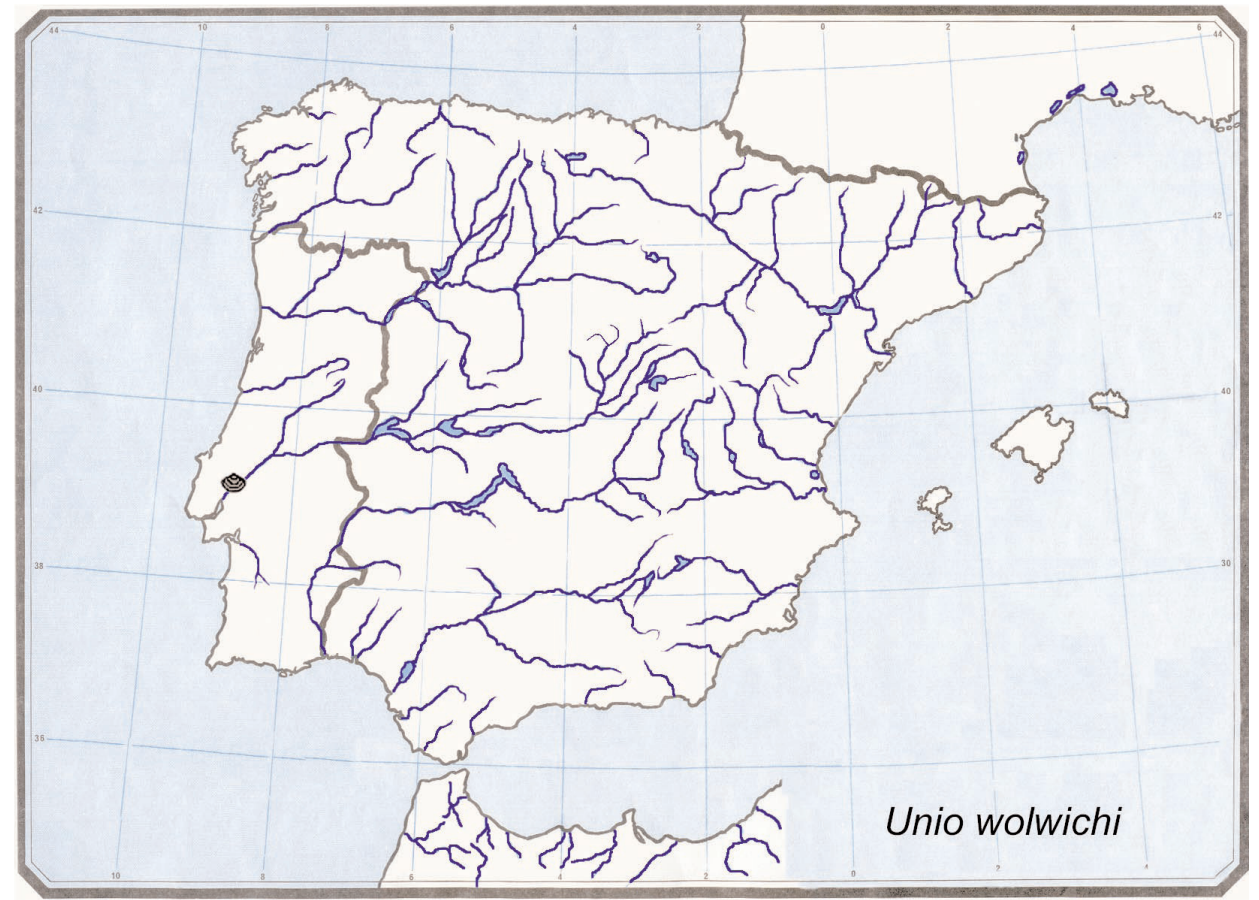

Fig. 3.- Distribución del material contenido en las Colecciones del MNCN de Unio wolwichi Morelet, 1845.

Fig. 3.- Distribution of the Unio wolwichi Morelet, 1845 material from the Spanish MNCN Collections.

Obs.: En la etiqueta antigua aparece como Unio requieni Michaud, 1831.

MNCN 15.07/3275: Albufera de Valencia; Valencia; Colección Ortiz de Zárate; SECO; 2 ej.

Obs.: En la etiqueta antigua aparece como Unio turtoni valentinus Rossmässler, 1854.

MNCN 15.07/3278: Río Ebro, Logroño; Logroño; Colección Ortiz de Zárate; SECO; 9 ej.

Obs.: Recogidas en las orillas, procedentes de aluviones, rodadas y algo estropeadas. En la etiqueta antigua aparece como Unio requieni Michaud, 1831.

MNCN 15.07/3290: Río Ebro, Cenicero; Logroño; Colección Ortiz de Zárate; SECO; 2 ej.

Obs.: En la etiqueta antigua aparece como Unio requieni Michaud, 1831.

MNCN 15.07/5215: Río Tajo, Presa de San Julián; Toledo; Colección J. Álvarez; 2/AGO/1962; SECO; 2 ej.

Obs.: Los ejemplares se encontraban junto con el lote MNCN 15.07/1791 $P$ littoralis y en la etiqueta antigua aparecen como Unio elongatulus Pfeiffer, 1825.

MNCN 15.07/5242: Río Bañuelo, embalse de Gasset, Fernancaballero; Ciudad Real; 13/NOV/1989; R. Araujo; SECO; 2 ej.

Obs.: Material de esta localidad ha sido estudiado por Nagel (2001), siendo adscrito mediante técnicas moleculares a la subespecie U. p. mancus. La morfología de la concha de los ejemplares que se conservan en el museo los hacen asignables a $U$. p. pictorum y en nada muestran caracteres morfológicos similares a los de las poblaciones de U. p. mancus de la península Ibérica. A pesar de ello incluimos este lote entre los pertenecientes a la subespecie U. p. mancus creada por Nagel (opus cit.) por recomendación del mencionado autor.

MNCN 15.07/5243: Albufera de Valencia; Valencia; SECO; 1 ej.

Obs.: En la etiqueta antigua aparece como Unio valentinus Rössmassler, 1854.

MNCN 15.07/5265: Canal de la Albufera, El Saler; Valencia; Colección Cobos; 26/JUL/1968; Gasull; SECO; 5 ej. Obs.: En la etiqueta antigua aparece como Unio valentinus Rossmässler.

MNCN 15.07/5267: Sin localidad; IFIE (Instituto Forestal de Investigaciones y Experimentación); SECO; 22 ej.

MNCN 15.07/5272: Río Miga, Vilanova de la Muga, cerca de Mas d'en Gaita; Gerona; Colección Ortiz de Zárate; AGO/1967; SECO; 3 ej.

Obs.: En la etiqueta antigua aparece como Unio turtoni aleroni Companyo y Massot.

MNCN 15.07/5273: Canal de la Albufera, El Saler; Valencia; Colección Cobos; 26/JUL/1968; Gasull; SECO; 3 ej.

Obs.: En la etiqueta antigua aparece como Unio valentinus Rossmässler. 


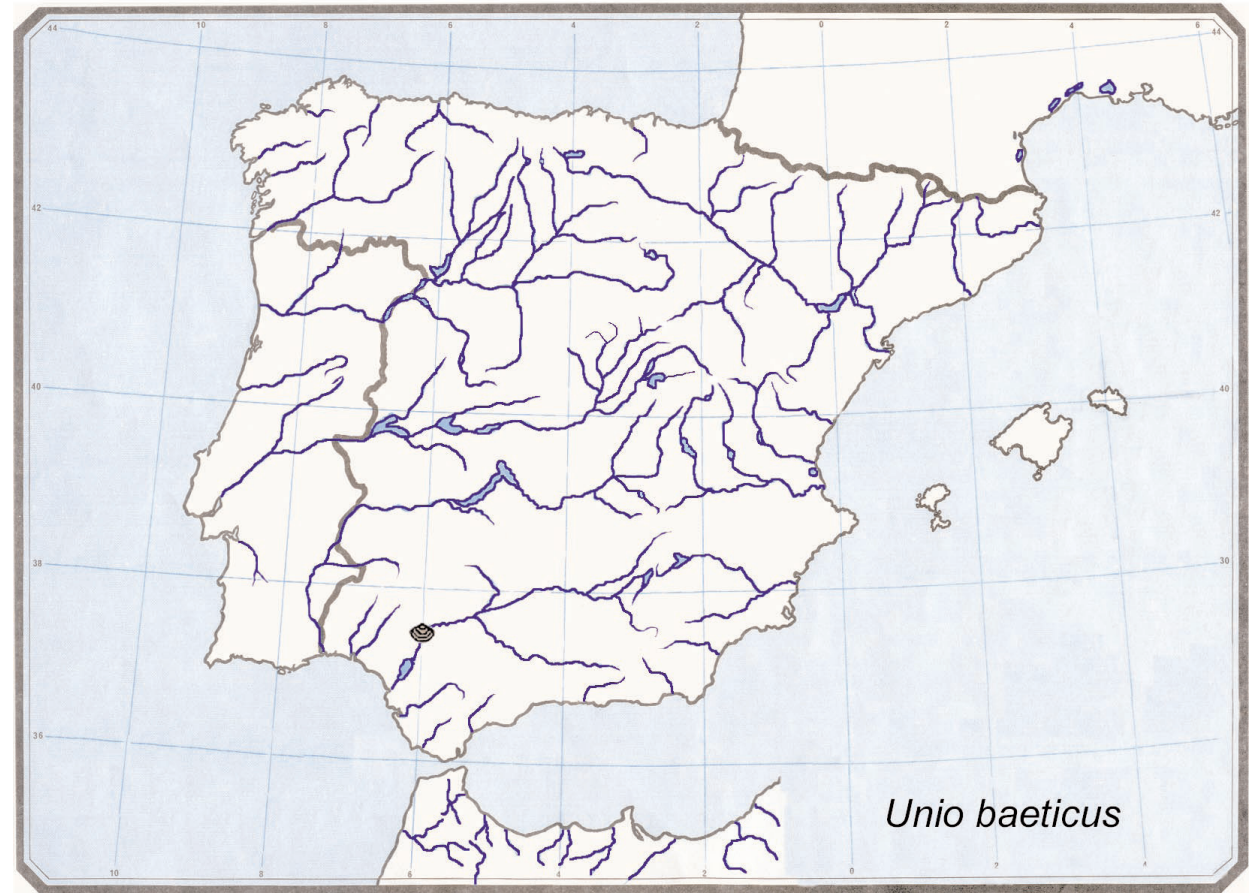

Fig. 4.- Distribución del material contenido en las Colecciones del MNCN de Unio baeticus Kobelt, 1887.

Fig. 4.- Distribution of the Unio baeticus Kobelt, 1887 material from the Spanish MNCN Collections.

\section{Ejemplares de Unio de difícil adscripción:}

\section{Unio wolwichi Morelet, 1845}

Unio wolwichi Morelet, 1845. Description des Moll. Terr. Et fluviat. Du Portugal, pág. 105, lám. XIII, fig. 1.

Esta especie fue descrita por Morelet (1845) con material recolectado por $\mathrm{M}$. Wolwich en el valle del Tajo, entre Villa-Nova y Azambuja (Portugal), treinta y dos años después el propio Morelet en "Moluscos terrestres y fluviales de Portugal" (Morelet, 1887) niega la presencia del $U$. wolwichi en la fauna de Portugal ya que sería una especie procedente de Australia. Posteriormente Bourguignat la señala como procedente de sudamérica pero tanto Locard (1899) y Simpson (1900) se niegan a la teoría de un origen exótico y su importación. Por último Haas (1917) llega a la conclusión de que $U$. wolwichi debe eliminarse de la fauna portuguesa para pasar a la sinonimia de Diplodon parallelopipedon (Lea, 1834) del río de la Plata. Esta última, es la opinión más aceptada, aunque Azpeitia (1933) menciona el enorme parecido de los ejemplares de Morelet con los de la especie $U$. auratus Swainson, cosa que hemos comprobado. Esperamos que en breve, el uso de técnicas de taxonomía molecular pongan fin a esta controversia.

MNCN 15.07/529: Portugal; Colección Hidalgo; SECO; 2 ej. Obs.: Lote con dos etiqueta antiguas, una de ellas posiblemente de Morelet. Material enviado a Hidalgo por el autor de la especie. En la etiqueta antigua aparece como Unio wolwichi Morelet, 1845. Material estudiado en Azpeitia (1933: 423,428)

MNCN 15.07/763: Portugal?; Colección Azpeitia 1148; SECO; 1 ej.

Obs.: Azpeitia indica que es uno de los ejemplares originales por los que se fundó la especie. Posible material tipo. En la etiqueta antigua aparece como Unio wolwichi Morelet, 1845. Material estudiado y figurado en Azpeitia (1933: 423,428, lám. X, fig. 59).

\section{Unio baeticus Kobelt, 1887}

MNCN 15.07/759: Sevilla ?; Sevilla; Colección Azpeitia 1166; SECO; 1 ej.

Obs.: En la etiqueta antigua aparece como Unio baeticus Kobelt, 1887. Material estudiado y figurado en Azpeitia (1933: 254-257, lám. IV, fig. 23). 
MNCN 15.07/1553: Sevilla ?; Sevilla; Colección Azpeitia 1166; SECO; 1 ej.

Obs.: En la etiqueta antigua aparece como Unio baeticus Kobelt, 1887. Material estudiado en Azpeitia (1933: 254-257).

\section{Subfamilia Quadrulinae Haas, 1929}

Género Potomida Swainson, 1840

Potomida littoralis (Lamarck, 1801)

Unio littoralis Lamarck, 1801, Syst. Anim. sans Vert.: 114.

Unio bigerrensis Millet, 1843.

Unio calderoni Kobelt, 1887.

Unio circinatus Drouët, 1893.

Unio gandiensis Drouët, 1888

Unio hispalensis Kobelt, 1887.

Unio rhysopygus Drouët, 1893.

Unio salvadori Westerlund, 1892

Unio subreniformis Bourguignat, 1863.

Unio subtetragonus Michaud, 1831.

Unio umbonatus Rossmässler, 1844.

Psilunio littoralis (Lamarck, 1801)

MNCN 15.07/219: España; 1954; SECO; 2 ej.

Obs.: En la etiqueta antigua aparece como Unio gandiensis Drouët, 1888

MNCN 15.07/221: Río Serpis, Gandía; Valencia; SECO; 2 ej. Obs.: En la etiqueta antigua aparece como Unio gandiensis Drouët, 1888.

MNCN 15.07/224: Río Júcar, Algemesí; Valencia; Colección Azpeitia 630; SECO; 12 ej.

Obs.: En la etiqueta antigua aparece como Unio circinatus Drouët, 1893. Material estudiado en Azpeitia (1933: 212-214).

MNCN 15.07/230: Río Nervión, Bilbao; Vizcaya; Colección Azpeitia 1155; Hidalgo; SECO; 1 ej.

Obs.: El ejemplar se encontraba en un lote de U. alero$n i$. En la etiqueta antigua aparece como Unio littoralis.

MNCN 15.07/239: Río Segura, Orihuela; Alicante; SECO; 1 ej. Obs.: En la etiqueta antigua aparece como Unio littoralis Lamarck.

MNCN 15.07/250: Río Miño, Tuy; Pontevedra; Colección Azpeitia 1155; SECO; 1 ej.

Obs.: En la etiqueta antigua aparece como Unio cameratus Drouët, 1893. Material estudiado y figurado en Azpeitia (1933: 365-366, lám. 8, fig. 47).

MNCN 15.07/264: Río Guadaira; Sevilla; Colección Azpeitia 1153; Calderón; SECO; 1 ej.

Obs.: En la etiqueta antigua aparece como Unio calderoni Kobelt, 1887. Material estudiado en Azpeitia (1933: 221-223).

MNCN 15.07/277: Canales de río Turia, Valencia; Valencia; Colección Azpeitia 632; SECO; 1 ej.

Obs.: En la etiqueta antigua aparece como Unio gandiensis Drouët, 1888. Material estudiado en Azpeitia (1933: 207-209).

MNCN 15.07/281: Río Zadorra; Álava; Colección Azpeitia 632; SECO; 1 ej.

Obs.: En la etiqueta antigua aparece como Unio gandiensis Drouët, 1888. Material estudiado en Azpeitia (1933: 207-209).
MNCN 15.07/296: Río Guadalquivir; Colección Azpeitia 635; SECO; 11 ej.

Obs.: Según Azpeitia siete de los ejemplares proceden de la colección Pérez Arcas. En la etiqueta antigua aparece como Unio hispalensis Kobelt, 1887. Material estudiado en Azpeitia (1933: 219-221.

MNCN 15.07/331: Torroella de Montgrí; Gerona; Colección Paz y Membiela; SECO; 2 ej.

Obs.: En la etiqueta antigua aparece como Unio littoralis Lamarck.

MNCN 15.07/337: Acequia de riego, río Jalón, Almunia de Doña Godina; Zaragoza; Colección Azpeitia 53; SECO; 3 ej.

Obs.: En la etiqueta antigua aparece como Unio littoralis Lamarck. Material estudiado en Azpeitia (1933: 185193).

MNCN 15.07/338: Torroella de Montgrí; Gerona; Colección Paz y Membiela; SECO; 1 ej.

Obs.: En la etiqueta antigua aparece como Unio littoralis Lamarck.

MNCN 15.07/339: Río Sió, Balaguer; Lérida; Colección Azpeitia 53; SECO; 1 ej.

Obs.: En la etiqueta antigua aparece como Unio littoralis Lamarck. Material estudiado en Azpeitia (1933: 185-193).

MNCN 15.07/341: Río Ayllón, Linares; Segovia; Colección Azpeitia 53; SECO; 2 ej.

Obs.: En la etiqueta antigua aparece como Unio littoralis Lamarck. Material estudiado en Azpeitia (1933: 185-193).

MNCN 15.07/343: Riera de Llémana, Bajo Ampurdán; Gerona; Colección Azpeitia 53; Chia; SECO; 1 ej. Obs.. En la etiqueta antigua aparece como Unio littoralis Lamarck. Material estudiado en Azpeitia (1933: 185-193).

MNCN 15.07/345: Río Ebro, Canal Imperial; Zaragoza; Colección Azpeitia 53; SECO; 1 ej.

Obs.: En la etiqueta antigua aparece como Unio littoralis Lamarck. Material estudiado en Azpeitia (1933: 185-193).

MNCN 15.07/347: Lagunas de Ruidera; Ciudad Real; Colección Azpeitia 53; Boscá; SECO; 1 ej.

Obs.: En la etiqueta antigua aparece como Unio littoralis Lamarck. Material estudiado en Azpeitia (1933: 185-193).

MNCN 15.07/349: Río Palmones; Cádiz; Colección Azpeitia 53; Gavala; SECO; 2 ej.

Obs.: En la etiqueta antigua aparece como Unio littoralis Lamarck. Material estudiado en Azpeitia (1933: 185-193).

MNCN 15.07/351: Río Guadalquivir, Cazorla; Jaén; Colección Azpeitia 53; T. Muro; SECO; 2 ej.

Obs.: En la etiqueta antigua aparece como Unio littoralis Lamarck. Material estudiado en Azpeitia (1933: 185-193).

MNCN 15.07/367: Río Segura, Orihuela; Alicante; Colección Azpeitia 1161; SECO; 2 ej.

Obs.: En la etiqueta antigua aparece como Unio pseudolittoralis Clessin, 1875

MNCN 15.07/384: Río Guadaira; Sevilla; Colección Azpeitia 1153; SECO; 1 ej.

Obs.: En la etiqueta antigua aparece como Unio calderoni ? Kobelt, 1887. Material estudiado en Azpeitia (1933: 221-223).

MNCN 15.07/388: Río Tajo, Badajoz; Badajoz; Colección Hidalgo; Paz; SECO; 1 ej.

MNCN 15.07/391: Río Guadalhorce, Málaga; Málaga; Colección Azpeitia 1147; Ruiz; SECO; 1 ej. 
Obs.: En la etiqueta antigua pone Unio salvadori (= hispalensis Kobelt) Westerlund. Material estudiado en Azpeitia (1933: 224-225).

MNCN 15.07/422: Laguna de Almenara; Castellón; Colección Azpeitia 631; SECO; 1 ej.

Obs.: En la etiqueta antigua aparece como Unio rhysopygus Drouët, 1893. Material estudiado y figurado en Azpeitia (1933: 225-227, lám. 3, fig. 16).

MNCN 15.07/426: Río Tajo; Toledo; Colección Azpeitia 634; SECO; 9 ej.

Obs.: En la etiqueta antigua aparece como Unio umbonatus Rossmässler, 1844. Material estudiado en Azpeitia (1933: 216-219).

MNCN 15.07/427: Río Guadaira; Sevilla; Colección Azpeitia 1147; SECO; 1 ej.

Obs.: En la etiqueta antigua aparece como Unio salvadori Westerlund, 1892. Material estudiado en Azpeitia (1933: 224-225).

MNCN 15.07/433: Río Guadaira; Sevilla; Colección Azpeitia 1147; SECO; 1 ej.

Obs.: En la etiqueta antigua aparece como Unio salvadori (= hispalensis Kobelt) Westerlund. Material estudiado en Azpeitia (1933: 224-225).

MNCN 15.07/434: Río Tajo; Toledo; Colección Azpeitia 634; SECO; 6 ej.

Obs.: En la etiqueta antigua aparece como Unio umbonatus Rossmässler, 1844. Material estudiado en Azpeitia (1933: 216-219).

MNCN 15.07/458: Caz en el río Jarama, Mejorada del Campo; Madrid; Colección Azpeitia 634; SECO; 1 ej.

Obs.: En la etiqueta antigua aparece como Unio umbonatus Rossmässler, 1844. Material estudiado en Azpeitia (1933: 216-219).

MNCN 15.07/461: Canales de río Turia, Valencia; Valencia; Colección Azpeitia 632; SECO; 3 ej.

Obs.: En la etiqueta antigua aparece como Unio gandiensis Drouët, 1888. Material estudiado en Azpeitia (1933: 207-209).

MNCN 15.07/463: Río Guadalquivir, Peñaflor; Sevilla; Colección Azpeitia 634; SECO; 1 ej.

Obs.: En la etiqueta antigua aparece como Unio umbonatus Rossmässler, 1844. Material estudiado en Azpeitia (1933: 216-219).

MNCN 15.07/465: Río Guadalquivir, Lora del Río; Sevilla; Colección Azpeitia 634; SECO; 1 ej.

Obs.: En la etiqueta antigua aparece como Unio umbonatus Rossmässler, 1844. Material estudiado en Azpeitia (1933: 216-219).

MNCN 15.07/468: España?; Colección Azpeitia 1161; Velasco; SECO; 1 ej.

Obs.: En la etiqueta antigua aparece como Unio pseudolittoralis variedad minor.

MNCN 15.07/469: España; Colección Azpeitia 634; SECO; 1 ej.

Obs.: En la etiqueta antigua aparece como Unio umbonatus Rossmässler, 1844.

MNCN 15.07/485: Río Serpis, Gandía; Valencia; Colección Azpeitia 632; SECO; 3 ej.

Obs.: En la etiqueta antigua aparece como Unio gandiensis Drouët, 1888. Material estudiado en Azpeitia (1933: 207-209).
MNCN 15.07/494: Río Guadalhorce; Málaga; Colección Azpeitia; SECO; 2 ej.

Obs.: En la etiqueta antigua aparece como Unio umbonatus Rossmässler, 1844. Material estudiado en Azpeitia (1933: 216-219).

MNCN 15.07/495: Acequia de Moncada; Valencia; Colección Azpeitia 634; SECO; 1 ej.

Obs.: En la etiqueta antigua aparece como Unio umbonatus Rossmässler, 1844. Material estudiado en Azpeitia (1933: 216-219).

MNCN 15.07/496: Valencia; Valencia; Colección Azpeitia; SECO; 3 ej.

Obs.: En la etiqueta antigua aparece como Unio umbonatus Rossmässler, 1844. Material estudiado en Azpeitia (1933: 216-219)

MNCN 15.07/507: "Aguas manantiales", Gandía; Valencia; Colección Azpeitia 632; Boscá; SECO; 2 ej.

Obs.: En la etiqueta antigua aparece como Unio gandiensis Drouët, 1888. Material estudiado en Azpeitia (1933: 207-209).

MNCN 15.07/514: Río Jarama; Colección Azpeitia; SECO; 1 ej. Obs.: Seguramente pertenece al lote MNCN 15.07/555 de San Martín de la Vega pues Azpeitia comenta que tiene tres ejemplares y en el lote había dos. En la etiqueta antigua aparece como Unio umbonatus Rossmässler, 1844. Material estudiado en Azpeitia (1933: 216-219).

MNCN 15.07/517: Río Guadalquivir; Colección Azpeitia 634; SECO; 2 ej.

Obs.: En la etiqueta antigua aparece como Unio umbonatus Rossmässler, 1844. Material estudiado en Azpeitia (1933: 216-219).

MNCN 15.07/531: Acequias de Almenara; Castellón; Colección Hidalgo; SECO; 4 ej.

Obs.: En la etiqueta antigua aparece como Acequias de Almenara, Valencia y aparece como Unio littoralis Lamarck, 1801.

MNCN 15.07/532: Río Ebro, La Zaida; Zaragoza; Colección Azpeitia 634; SECO; 2 ej

Obs.: En la etiqueta antigua aparece como Unio umbonatus Rossmässler, 1844. Material estudiado en Azpeitia (1933: 216-219)

MNCN 15.07/547: Desembocadura del Guadiana?; Huelva; Colección Azpeitia 634; SECO; 2 ej.

Obs.: En la etiqueta aparece como Unio umbonatus Rossmässler, 1844.

MNCN 15.07/555: Río Jarama, San Martín de la Vega; Madrid; Colección Azpeitia 634; SECO; 2 ej.

Obs.: En la etiqueta antigua aparece como Unio littoralis Rossmässler, 1844. Material estudiado en Azpeitia (1933: 216-219)

MNCN 15.07/559: Valencia; Colección Azpeitia 634; SECO; 1 ej. Obs.: En la etiqueta antigua aparece como Unio umbonatus Rossmässler, 1844.Material estudiado en Azpeitia (1933: 216-219).

MNCN 15.07/562: Río Guadalquivir; Córdoba; Colección Azpeitia 634; SECO; 2 ej.

Obs.: En la etiqueta antigua aparece como Unio umbonatus Rossmässler, 1844. Material estudiado en Azpeitia (1933: 216-219).

MNCN 15.07/568: Río Tajo, Aranjuez; Madrid; Colección Azpeitia 634; SECO; 3 ej. 
Obs.: En la etiqueta antigua aparece como Unio umbonatus Rossmässler, 1844. Material estudiado en Azpeitia (1933: 216-219).

MNCN 15.07/575: Río Genil, Loja; Granada; Colección Azpeitia 634; SECO; 1 ej.

Obs.: El ejemplar lleva otra etiqueta de Drouët. En la etiqueta antigua aparece como Unio umbonatus Rossmässler.

MNCN 15.07/585: Río Guadalquivir, Sevilla; Sevilla; Colección Azpeitia 634; SECO; 2 ej.

Obs.: En la etiqueta antigua aparece como Unio umbonatus Rossmässler, 1844. Material estudiado en Azpeitia (1933: 216-219).

MNCN 15.07/586: Río Tajo, Toledo; Toledo; Colección Azpeitia 1161; Martínez; SECO; 1 ej.

Obs.: En la etiqueta antigua aparece como Unio pseudolittoralis Clessin, 1875

MNCN 15.07/755: "Aguas manantiales", Gandía; Valencia; Colección Azpeitia 632; Boscá; SECO; 2 ej.

Obs.: En la etiqueta antigua aparece como Unio gandiensis Drouët, 1888. Material estudiado en Azpeitia (1933: 207-209).

MNCN 15.07/761: Río Jarama, puente de Arganda, Arganda; Madrid; Colección Hidalgo; 14/FEB/1897; SECO; 1 ej. Obs.: Asociado con dudas a la colección Hidalgo. La etiqueta lleva el número 3. En la etiqueta antigua aparece como Unio littoralis Cuvier.

MNCN 15.07/771: Río Miño, Tuy; Pontevedra; Colección Azpeitia 629; SECO; 1 ej.

Obs.: En la etiqueta antigua aparece como Unio bigerrensis; Millet de la Turtaudière, 1843. Material estudiado en Azpeitia (1933: 210-212).

MNCN 15.07/787: Laguna de Almenara; Castellón; Colección Azpeitia 631; SECO; 9 ej.

Obs.: En la etiqueta antigua aparece como Unio rhysopygus; Drouët, 1893. Material estudiado en Azpeitia (1933: 225-227).

MNCN 15.07/1445: Riera de Llaumona; Gerona; Colección Hidalgo; Chia; SECO; 3 ej.

Obs.: En la etiqueta antigua aparece como Unio subreniformis Bourguignat, 1864.

MNCN 15.07/1446: Río Guadalquivir, Sevilla; Sevilla; 1888; SECO; 2 ej.

Obs.: En la etiqueta antigua aparece como Unio hispalensis Kobelt, 1887.

MNCN 15.07/1457: Río Boco, Aveiro; Portugal. JUL/1879; SECO; 2 ej.

Obs.: En la etiqueta antigua aparece como Unio hispalensis Kobelt, 1887

MNCN 15.07/1463: Sevilla; SECO; 3 ej.

Obs.: En la etiqueta antigua aparece como Unio hispalensis Kobelt, 1887.

MNCN 15.07/1464: Lago de Bañolas; Gerona; Colección Hidalgo; Martorell; SECO; 3 ej.

Obs.: En la etiqueta antigua aparece como Unio subreniformis Bourguignat, 1864.

MNCN 15.07/1485: Pamplona; Navarra; SECO; 2 ej.

MNCN 15.07/1492: Río Ebro; Boscá; SECO; 32 ej.

Obs.: El lote tiene otra etiqueta que pone Uno, República Argentina.

MNCN 15.07/1551: Lago de Bañolas; Gerona; Colección Azpeitia 633; SECO; 15 ej.
Obs.: En la etiqueta antigua aparece como Unio subreniformis Bourguignat, 1864. Material estudiado en Azpeitia (1933: 227-231)

MNCN 15.07/1552: España?; SECO; 1 ej.

Obs.: En un lote de U. Aleroni (MNCN 15.07/779).

MNCN 15.07/1578: Río Tajo, Villarrubia de Santiago; Toledo; 1959; SECO; 28 ej.

MNCN 15.07/1582: Río Guadalquivir, presa de Son?; SECO; 8 ej.

MNCN 15.07/1584: Río Guadamez, Vega de los Maderos, Don Benito; Badajoz; Verano/1941; Bayón; SECO; 18 ej.

MNCN 15.07/1655: Lago de Bañolas; Gerona; SECO; 1 ej.

MNCN 15.07/1656: Gandía; Valencia; Colección Paz y Membiela; SECO; 1 ej.

Obs.: En la etiqueta antigua aparece como Unio littoralis.

MNCN 15.07/1677: Río Guadamez, Vega de los Maderos, Don Benito; Badajoz; Verano/1941; Bayón; SECO; 14 ej.

MNCN 15.07/1679: Río Tajo, Villarrubia de Santiago; Toledo; SECO; 5 ej.

Obs.: En la etiqueta antigua aparece como Unio hispalensis Kobelt, 1887.

MNCN 15.07/1704: Río Segura y río Júcar; SECO; 6 ej.

Obs.: El lote tiene varias etiquetas donde pone Segura y Valencia.

MNCN 15.07/1711: España?; SECO; 7 ej.

Obs.: En la etiqueta antigua aparece como Unio subtetragonus Michaud, 1831.

MNCN 15.07/1741: Lagunas de Ruidera; Ciudad Real; SECO; 2 ej.

MNCN 15.07/1743: Río Segura, Orihuela; Alicante; Colección Hidalgo; SECO; 3 ej.

Obs.: En la etiqueta antigua aparece como Unio umbonatus Rossmässler, 1844. Material citado en Azpeitia (1933: 216-219).

MNCN 15.07/1748: Lago de Bañolas; Gerona; Colección Azpeitia 633; SECO; 11 ej.

Obs.: En la etiqueta antigua aparece como Unio subreniformis Bourguignat, 1864. Material estudiado en Azpeitia (1933: 227-231).

MNCN 15.07/1749: Río Ebro, Canal Imperial de Aragón; Zaragoza; SECO; 22 ej.

Obs.: Uno de los ejemplares lleva el una etiqueta que pone $n^{\circ} 391$, Cabanes, Ampurdán.

MNCN 15.07/1777: Río Tiétar; Toledo; Colección J. Álvarez; SECO; 4

Obs.: En la etiqueta antigua aparece como Unio umbonatus Rossmässler, 1844.

MNCN 15.07/1785: Río Tajo; SECO; 1 ej. Obs.: Lote con una etiqueta antigua que lleva letra de Hidalgo.

MNCN 15.07/1788: Madrid; Madrid; SECO; 1 ej. Obs.: En la etiqueta antigua aparece como Unio littoralis Lamarck, 1801 variedad romboides.

MNCN 15.07/1791: Río Tajo, Presa de San Julián; Toledo; Colección J. Álvarez; 2/AGO/1962; SECO; 13 ej.

MNCN 15.07/1792: Río Tajo, Villarrubia de Santiago; Toledo; Colección J. Álvarez; 24/MAR/1961; SECO; 6 ej. Obs.: En la etiqueta antigua aparece como Unio umbonatus Rossmässler, 1844. 


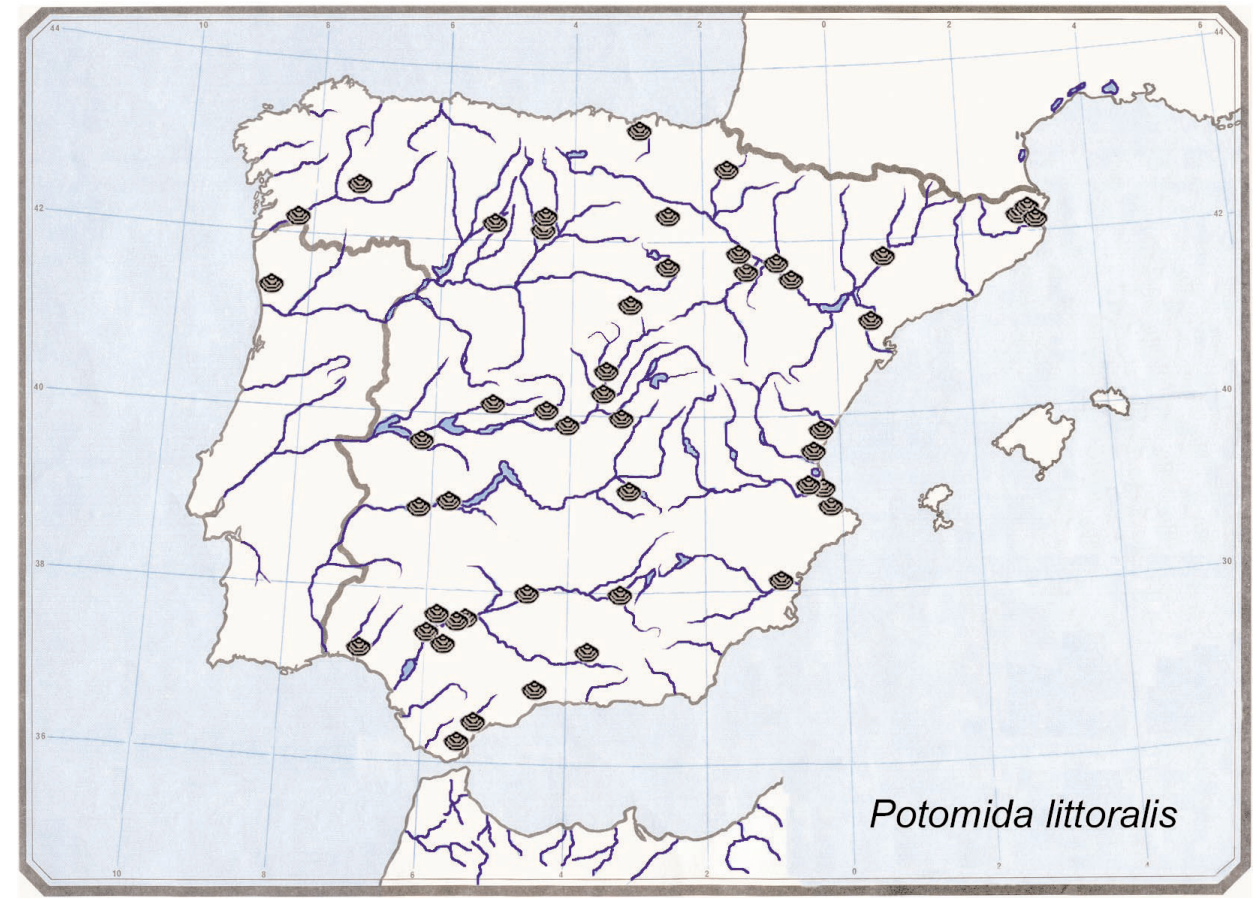

Fig. 5.- Distribución del material contenido en las Colecciones del MNCN de Potomida littoralis (Lamarck, 1801).

Fig. 5.- Distribution of the Potomida littoralis (Lamarck, 1801) material from the Spanish MNCN Collections.

MNCN 15.07/1793: Río Guadalquivir; Colección J. Álvarez; 16/JUN/1961; SECO; 2 ej.

Obs.: En la etiqueta antigua aparece como Unio littoralis Lamarck, 1801.

MNCN 15.07/1794: Río Jarama, puente de Arganda, Arganda; Madrid; Colección J. Álvarez; 22/ABR/1961; SECO; 1 ej. Obs.: En la etiqueta antigua aparece como Unio umbonatus Rossmässler, 1844

MNCN 15.07/1801: Río Tajo, Villarrubia de Santiago; Toledo; Colección J. Álvarez; 24/MAR/1961; SECO; 17 ej. Obs.: En la etiqueta antigua aparece como Unio umbonatus Rossmässler, 1844.

MNCN 15.07/1805: Río Guadamez, Vega de los Maderos, Don Benito; Badajoz; Verano/1941, SECO; 8 ej.

MNCN 15.07/1863: Río Guadamez, Vega de los Maderos, Don Benito; Badajoz; Verano/1941; Bayón; SECO; 5 ej.

MNCN 15.07/3260: Río Ebro, Mora de Ebro; Tarragona; Colección Ortiz de Zárate; AGO/1964; J. Auba (Leg); SECO; 2 ej.

MNCN 15.07/3268: Nájera; Rioja; Colección Ortiz de Zárate $\mathrm{n}^{\circ}$ 3; A. Ortiz de Zárate; SECO; 1 ej.

Obs.: En la etiqueta antigua aparece como Psilunio littoralis (Lamarck).

MNCN 15.07/3276: Canal de Castilla, Río Ucieza, Piña de Campos; Palencia; Colección Ortiz de Zárate; AGO/1954; Altimira; SECO; 1 ej.

MNCN 15.07/3277: Canal de Castilla, Río Ucieza, Piña de Campos; Palencia; Colección Ortiz de Zárate; AGO/1954; Altimira; SECO; 1 ej.
MNCN 15.07/3283: Valencia; Valencia; Colección Ortiz de Zárate $\mathrm{n}^{\circ} 1431$; SECO; 1 ej.

Obs.: En la etiqueta antigua aparece como Psilunio littoralis (Lamarck).

MNCN 15.07/3284: Río Duero, Soria; Soria; Colección Ortiz de Zárate; L. Martínez Rocandio; SECO; 1 ej. Obs.: En la etiqueta antigua aparece como Unio moquinianus cameratus Drouët, 1893.

MNCN 15.07/3287: Río Ebro?, Nájera; Rioja; Colección Ortiz de Zárate; SECO; 1 ej.

Obs.: En la etiqueta antigua aparece como Psilunio littoralis (Lamarck). Suponemos que la localidad es río Najerilla, ya que el Ebro no pasa por Nájera.

MNCN 15.07/3288: Río Júcar, Cullera; Valencia; Colección Ortiz de Zárate; Siro de Fez; SECO; 1 ej.

Obs.: En la etiqueta antigua aparece como Psilunio littoralis (Lamarck).

MNCN 15.07/3666: Canal Imperial de Aragón; Zaragoza; IFIE (Instituto Forestal de Investigaciones y Experimentación); MAR/1946; SECO; 2 ej.

Obs.: En la etiqueta antigua aparece como Psilunio littoralis.

MNCN 15.07/3709: Río Alberche, Embalse de Cazalegas; Toledo; Colección Leo Imperatori; SECO; 4 ej.

MNCN 15.07/5135: Río Tajo, Alcántara; Cáceres; Colección O. De Zárate; 27/AGO/1963; SECO; 2 ej.

Obs.: Ejemplares capturados bajo el puente de Alcántara. "Depositada en la parte más alta del límite de las grandes avenidas en la arena de la orilla". 
MNCN 15.07/5227: Río Guadiana, Mérida; Badajoz; IFIE (Instituto Forestal de Investigaciones y Experimentación); 21/JUN/1942; SECO; 16 ej.

Obs.: En la etiqueta antigua aparece como Psilunio littoralis.

MNCN 15.07/5232: Río Guadarranque; Cádiz; Colección Cobos; F. Ramírez; SECO; 2 ej.

MNCN 15.07/5233: Río Ebro, Villafranca del Ebro; Tarragona; Colección Cobos; C. Altimira; SECO; 2 ej. Obs.: En la etiqueta antigua aparece como Psilunio littoralis (Cuvier).

MNCN 15.07/5234: Río Sió, Balaguer; Lérida; Colección Cobos (ex -coll Altimira); AGO/1961; SECO; 2 ej. Obs.: En la etiqueta antigua aparece como Psilunio littoralis (Cuvier).

MNCN 15.07/5235: Río Guadarranque, San Roque; Cádiz; Colección Cobos; MAY/1965; F. Ramírez; SECO; 1 ej. Obs.: En la etiqueta antigua aparece como Psilunio littoralis (Cuvier)

MNCN 15.07/5236: Río Cea, Valdescorriel; Zamora; Colección Cobos; 30/AGO/1978; L. Romero; SECO; 2 ej.

Obs.: En la etiqueta antigua aparece como Psilunio littoralis (Cuvier).

MNCN 15.07/5237: Río Viar, puente en la carretera de Burguillo; Sevilla; Colección Cobos; MAY/1965; L. García; SECO; 1

Obs.: En la etiqueta antigua aparece como Psilunio $s p$.

MNCN 15.07/5238: Río Ebro, Mora de Ebro; Tarragona; Colección Cobos; AGO/1964; Auba; SECO; 2 ej.

Obs.: Material procedente de la colección de C Altimira, legado en 1965 a Cobos. En la etiqueta antigua aparece como Psilunio littoralis (Cuvier).

MNCN 15.07/5239: Río Cabe, Monforte de Lemos; Lugo; Colección Cobos; Álvarez; SECO; 4 ej.

MNCN 15.07/5240: Río Guadalquivir, La Iruela; Jaén; Colección Cobos; AGO/1975; SECO; 11 ej.

MNCN 15.07/5241: Río Guadiaro; Colección Cobos; F. Ramírez; SECO; 2 ej.

Obs.: El material es del río Guadiaro en Málaga o Cádiz.

MNCN 15.07/5245: Don Benito; Badajoz; SECO; 5 ej.

MNCN 15.07/5255: “Acequia de ¿Hernando?”, Santomera; Murcia; IFIE (Instituto Forestal de Investigaciones y Experimentación); SECO; 3 ej. Obs.: La fecha de captura es Mayo de 194 ?

MNCN 15.07/5260: Río Carrión, Palencia; Palencia; IFIE (Instituto Forestal de Investigaciones y Experimentación); 20/ABR/1945; SECO; 2 ej. Obs.: En la etiqueta antigua aparece como Unio littoralis Cuvier.

MNCN 15.07/5261: Río Jarama, San Fernando de Henares; Madrid; IFIE (Instituto Forestal de Investigaciones y Experimentación); 12/AGO/1943; SECO; 14 ej. Obs.: En la etiqueta antigua aparece como Psilunio littoralis.

Subfamilia Anodontinae Ortmann, 1912

Género Anodonta Lamarck, 1799

Anodonta cygnea (Linnaeus, 1758)

Mytilus cygneus Linnaeus, 1758. Systema Naturae, Editio Decima, Tomo I, pag. 706, num. 218.
Anodonta anatina (Linnaeus, 1758).

Anodonta baetica Kobelt, 1887.

Anodonta bicolor Drouët, 1893.

Anodonta calderoni Kobelt, 1887.

Anodonta castroi Bourguignat, 1881.

Anodonta cellensis (Gmelin in Linnaeus, 1791).

Anodonta emacerata Drouët, 1893.

Anodonta embiella Hagenmüller in Castro, 1883.

Anodonta glaucina Drouët, 1893.

Anodonta latirostris Drouët, 1893.

Anodonta lusoiana Castro, 1883.

Anodonta melinia Bourguignat, 1865.

Anodonta mollis Drouët, 1893.

Anodonta nobilis Drouët, 1893.

Anodonta piscinalis Nilsson, 1822.

Anodonta prasina Drouët, 1893.

Anodonta submacilenta Servain, 1880.

Anodonta valentina Drouët, 1893

Anodonta ventricosa Pfeiffer, 1825.

Anodonta viriata Servain, 1880

Anodonta wenceslai Castro, 1883.

MNCN 15.07/396: Albufera de Valencia; Valencia; Colección Azpeitia; SECO; 1 ej.

MNCN 15.07/627: Albufera de Valencia; Valencia; Colección Hidalgo; SECO; 5 ej.

Obs.: En la etiqueta antigua aparece como Anodonta anatina (Linnaeus, 1758).

MNCN 15.07/629: Dehesa al N del Paso, Albufera de Valencia; Valencia; SECO; 3 ej.

Obs.: En la etiqueta antigua aparece como Anodonta cygnea Linnaeus.

MNCN 15.07/637: Albufera de Valencia; Valencia; Colección Azpeitia 1172; SECO; 2 ej.

Obs.: En la etiqueta antigua aparece como Anodonta castroi Bourguignat, 1881. Estudiado en Azpeitia (1933: 539-542).

MNCN 15.07/639: Arroyo Gabino, Alcolea del Río; Sevilla; Colección Azpeitia 1571; Gavala; SECO; 1 ej.

Obs.: En la etiqueta antigua aparece como Anodonta calderoni Pfr.

MNCN 15.07/648: Entre la Mata del Fanch y la Mateta de la Sequieta, Albufera de Valencia; Valencia; SECO; 3 ej. Obs.: Material recolectado en arenas fangosas entre 1,25 y 1,5 metros.

MNCN 15.07/650: Río Palmones; Cádiz; Colección Azpeitia 704; SECO; 1 ej.

Obs.: En la etiqueta antigua figura como $A$ cellensis (= zellensis Gmelin in Linnaeus, 1791).

MNCN 15.07/658: Dehesa entre la Mata del Fanch y Mateta de la Sequieta, Albufera de Valencia; Valencia; SECO; 3 ej. Obs.: Material recolectado en arenas entre 0 y $0,5 \mathrm{~m}$.

MNCN 15.07/660: Dehesa entre Mata del Fanch y Mateta de la Sequieta, Albufera de Valencia; Valencia; SECO; 4 ej. Obs.: Material recolectado en arena entre 0,75 y 1 metro de profundidad.

MNCN 15.07/664: Albufera de Valencia; Valencia; Colección Azpeitia 1162; Azpeitia; SECO; 1 ej.

Obs.: En la etiqueta antigua aparece como Anodonta bicolor Drouët, 1893. Estudiado en Azpeitia (1933: 528-529).

MNCN 15.07/668: Valencia; Valencia; Colección Hidalgo; SECO; 1 ej.

Obs.: Lote con dos etiquetas antiguas que escritas en francés indican Anodonta adusta Drouët. Unique. 
Hidalgo. ¿Material tipo?. En la etiqueta antigua aparece como Anodonta adusta Drouët, 1893.

MNCN 15.07/670: Albufera de Valencia; Valencia; Colección Azpeitia 1162; Azpeitia; SECO; 1 ej.

Obs.: En la etiqueta antigua aparece como Anodonta bicolor Drouët, 1893. Estudiado en Azpeitia (1933: 528529).

MNCN 15.07/674: Paso entre la Mata del Fanch y Petillet, Albufera de Valencia; Valencia; SECO; 3 ej.

Obs.: Material recolectado en fango entre 0.2 y 0.4 metros.

MNCN 15.07/676: Entrada sur del Paso, Albufera de Valencia; Valencia; SECO; 2 ej.

Obs.: Material recolectado en banco de fango a 0,2 metros de profundidad.

MNCN 15.07/677: Albufera de Valencia; Valencia; Colección Azpeitia 1162; Azpeitia; SECO; 1 ej.

Obs.: En la etiqueta antigua aparece como Anodonta bicolor Drouët, 1893. Material estudiado y figurado en Azpeitia (1933: 528-529, lám. XX, fig. 79).

MNCN 15.07/685: Albufera de Valencia; Valencia; Colección Hidalgo; SECO; 2 ej.

Obs.: La etiqueta antigua no se parece a las de la colección Hidalgo y en ella aparece como Anodonta piscinalis Nilsson, 1822.

MNCN 15.07/687: Albufera de Valencia; Valencia; Colección Azpeitia 676; SECO; 2 ej.

Obs.: En la etiqueta antigua aparece como Anodonta melinia Bourguignat, 1865. Material estudiado y figurado en Azpeitia (1933: 533-538, lám. XXIV, fig. 86).

MNCN 15.07/691: Albufera de Valencia; Valencia; Colección Azpeitia 676; SECO; 1 ej.

Obs.: En la etiqueta antigua aparece como Anodonta melinia Bourguignat, 1865. Material estudiado en Azpeitia (1933: 533-538).

MNCN 15.07/693: Galicia; Colección Azpeitia 1164; Pérez Arcas; SECO; 2

Obs.: En la etiqueta antigua aparece como Anodonta prasina Drouët, 1893.

MNCN 15.07/718: Río Javalón, Granatula de Calatrava; Ciudad Real; Colección Azpeitia 1163; SECO; 2 ej.

Obs.: Las etiquetas de Azpeitia indican que Anodonta lusoiana Castro 1883 y Anodonta glaucina Drouët, 1893 son la misma especie. En la etiqueta antigua aparece como Anodonta lusoiana; Castro, 1883. Material estudiado y figurado en Azpeitia (1933: 567-569, lám. XXVII, fig.94).

MNCN 15.07/730: Albufera de Valencia; Valencia; Colección Azpeitia 1171; SECO; 5 ej.

Obs.: En la etiqueta antigua aparece como Anodonta emacerata Drouët, 1893.

MNCN 15.07/732: Albufera de Valencia; Valencia; Colección Azpeitia 1171; SECO; 2 ej.

Obs.: En la etiqueta antigua aparece como Anodonta emacerata Drouët, 1893. Material estudiado en Azpeitia (1933: 544)

MNCN 15.07/736: Albufera de Valencia; Valencia; Colección Azpeitia 1171; SECO; 2 ej.

Obs.: En la etiqueta antigua aparece como Anodonta emacerata Drouët, 1893

MNCN 15.07/738: Albufera de Valencia; Valencia; Colección Azpeitia 1171; SECO; 2 ej.
Obs.: En la etiqueta antigua aparece como Anodonta emacerata Drouët, 1893. Material estudiado y figurado en Azpeitia (1933: 544; lám. XXV, fig. 90).

MNCN 15.07/740: Río Mondego; Portugal; Colección Azpeitia 1181; Paz; SECO; 1 ej.

Obs.: En la etiqueta antigua aparece como Anodonta embiella Hagenmüller, in Castro, 1883. Material estudiado y figurado en Azpeitia (1933: 594-596, lám. XXX, fig. 101).

MNCN 15.07/762: Extremadura; Colección Hidalgo; Graells; SECO; 2 ej.

Obs.: En la etiqueta antigua aparece como Anodonta latirostris Drouët, 1893.

MNCN 15.07/768: Extremadura; Colección Azpeitia 1334; Graells; SECO; 2 ej.

Obs.: En la etiqueta antigua aparece como Anodonta latirostris Drouët, 1893.

MNCN 15.07/772: Extremadura; Colección Azpeitia 1334; SECO; 1 ej.

MNCN 15.07/778: Albufera de Valencia; Valencia; Colección Azpeitia 676; SECO; 1 ej.

Obs.: En la etiqueta antigua aparece como Anodonta melinia Bourguignat, 1865. Material estudiado en Azpeitia (1933: 533-538).

MNCN 15.07/780: Albufera de Valencia; Valencia; Colección Azpeitia 676; SECO; 1 ej.

Obs.: En la etiqueta antigua aparece como Anodonta melinia Bourguignat, 1865. Material estudiado en Azpeitia (1933: 533-538).

MNCN 15.07/784: Albufera de Valencia; Valencia; Colección Azpeitia 676; SECO; 1 ej.

Obs.: En la etiqueta antigua aparece como Anodonta melinia Bourguignat, 1865. Material estudiado en Azpeitia (1933: 533-538)

MNCN 15.07/786: Albufera de Valencia; Valencia; Colección Azpeitia 676; SECO; 1 ej.

Obs.: En la etiqueta antigua aparece como Anodonta melinia Bourguignat, 1865. Material estudiado en Azpeitia (1933: 533-538).

MNCN 15.07/794: Albufera de Valencia; Valencia; Colección Azpeitia 506; SECO; 1 ej.

Obs.: En la etiqueta antigua aparece como Anodonta nobilis Drouët, 1893. Material estudiado y figurado en Azpeitia (1933: 530-531, lám. XXII, fig. 82).

MNCN 15.07/796: Río Miño, Tuy; Pontevedra; Colección Azpeitia 1576; SECO; 1 ej.

Obs.: En la etiqueta antigua aparece como Anodonta wenceslai Castro, 1883. Material estudiado y figurado en Azpeitia (1933: 572-575, lám. XXVII, fig. 96).

MNCN 15.07/804: Albufera de Valencia; Valencia; Colección Azpeitia; SECO; 2 ej.

Obs.: En la etiqueta antigua aparece como Anodonta viriata Servain, 1880.

MNCN 15.07/806: Albufera de Valencia; Valencia; Colección Azpeitia 1574; SECO; 1 ej.

Obs.: En la etiqueta antigua aparece como Anodonta viriata Servain, 1880. Material estudiado y figurado en Azpeitia (1933: 524-527, lám. XIX, fig. 77).

MNCN 15.07/817: Albufera de Valencia; Valencia; Colección Azpeitia 1170; SECO; 1 ej. 
Obs.: En la etiqueta antigua aparece como Anodonta valentina Drouët, 1893. Material estudiado en Azpeitia (1933: 542-543).

MNCN 15.07/819: Albufera de Valencia; Valencia; Colección Azpeitia 1170; SECO; 1 ej.

Obs.: Ejemplar bastante deteriorado. En la etiqueta antigua aparece como Anodonta valentina; Drouët, 1893. Material estudiado en Azpeitia (1933: 542-543).

MNCN 15.07/821: Albufera de Valencia; Valencia; Colección Azpeitia 1170; SECO; 1 ej.

Obs.: En la etiqueta antigua aparece como Anodonta valentina Drouët, 1893. Material estudiado en Azpeitia (1933: 542-543).

MNCN 15.07/827: Arroyo Gabino, entre Alcolea del Río y Villaverde del Río; Sevilla; Colección Azpeitia 931; Gavala; SECO; 1 ej.

Obs.: En la etiqueta antigua aparece como Anodonta ventricosa; Pfeiffer, 1825. Material estudiado en Azpeitia (1933: 472-477).

MNCN 15.07/831: Arroyo Gabino, entre Alcolea del Río y Villaverde del Río; Sevilla; Colección Azpeitia 931; Gavala; SECO; 1 ej.

Obs.: En la etiqueta antigua aparece como Anodonta ventricosa; Pfeiffer, 1825. Material estudiado en Azpeitia (1933: 472-477).

MNCN 15.07/837: Albufera de Valencia; Valencia; Colección Azpeitia 1574; SECO; 2 ejs.

Obs.: En la etiqueta antigua aparece como Anodonto viriata Servain, 1880. Material estudiado en Azpeitia (1933: 524-527).

MNCN 15.07/843: Albufera de Valencia; Valencia; Colección Azpeitia 1575; SECO; 2 ej.

Obs.: En la etiqueta antigua aparece como Anodonta submacilenta Servain, 1880. Material estudiado y figurado en Azpeitia (1933: 515-520, lám. XVI, fig. 73).

MNCN 15.07/851: Albufera de Valencia; Valencia; Colección Azpeitia 1170; SECO; 1 ej.

Obs.: En la etiqueta antigua aparece como Anodonta valentina Drouët, 1893. Material estudiado en AZPEITIA (1933: 542-543).

MNCN 15.07/855: Albufera de Valencia; Valencia; Colección Azpeitia 1170; SECO; 3 ej.

Obs.: Ejemplares bastante deteriorados. En la etiqueta antigua aparece como Anodonta valentina Drouët, 1893. Material estudiado en Azpeitia (1933: 542-543).

MNCN 15.07/1447: Albufera de Valencia; Valencia; SECO; 1 ej. Obs.: Lote con dos etiqueta antiguas, una de ellas pone Clóchina, Albufera de Valencia.

MNCN 15.07/1452: Río Oñar, Gerona; SECO; 2 ej. Obs.: En la etiqueta antigua pone Anodonta glaucina Drouët.

MNCN 15.07/1538: Dehesa al N del Paso, Albufera de Valencia; Valencia; SECO; 1 ej.

Obs.: Material recolectado en arena fangosa a 1-1.5 metros de profundidad. En la etiqueta antigua aparece como Anodonta cygnea $\mathrm{L}$.

MNCN 15.07/1543: Banco de arena a la entrada norte del Paso, Albufera de Valencia; Valencia; SECO; 2 ej.

MNCN 15.07/1558: Extremidad Norte de la Dehesa, salida del Carrer del Saler, Albufera de Valencia; SECO; 1 ej. Obs.: Material recolectado en fango arenoso, 0,3-0,5 metros de profundidad.
MNCN 15.07/1579: Rincón entre Botillet y Dehesa, Albufera de Valencia; Valencia; SECO; 8 ej.

Obs: Material recolectado en fango entre 0 y 0,5 metros de profundidad.

MNCN 15.07/1581: Dehesa a 200 metros de su extremidad N, Albufera de Valencia; Valencia; SECO; 2 ej.

Obs.: Material recolectado en fango líquido entre 0.2 y 0.3 metros.

MNCN 15.07/1583: Puerto de Silla, Albufera de Valencia; Valencia; SECO; 1 ej.

Obs.: En fango de humus entre 0.2 y $0.3 \mathrm{~m}$ de profundidad. En la etiqueta antigua aparece como Anodonta cygnea L.

MNCN 15.07/1587: Lagunas de Almenara; Castellón; SEP/1902; SECO; 3 ej.

MNCN 15.07/1588; Río Guadaira; Sevilla; SECO; 1 ej. Obs.: En la etiqueta antigua aparece como Anodonta baetica Kobelt, 1887.

MNCN 15.07/1589; Carrer del Saler, Albufera de Valencia; Valencia; SECO. Varias valvas diferentes rotas.

Obs.: Ejemplares bastante deteriorados.

MNCN 15.07/1591: Albufera de Valencia; Valencia; SECO; 1 ej.

MNCN 15.07/1622: Río Palmones; Cádiz; Colección Azpeitia 704; Gavala; SECO; 1 ej.

Obs.: En la etiqueta antigua aparece como Anodonta cellensis Schröter $=$ Anodonta cygnea $($ Linnaeus, 1758). Estudiado y figurado en Azpeitia (1933: 468-472, lám. XIV, fig. 68)

MNCN 15.07/1624: Dehesa al norte del Paso, Albufera de Valencia; Valencia; SECO; 3 ej.

Obs.: Material recolectado en arenas entre 0.54 y 1 metros.

MNCN 15.07/1775: Río Tiétar, Buenaventura; Toledo; Colección Álvarez; 10/AGO/1976; A. Valledor; SECO; 2 ej.

MNCN 15.07/3264: Canal de Catarroja, Albufera de Valencia; Valencia; Colección Ortiz de Zárate; SECO; 1 ej. Obs.: En fango de humus entre 0,2 y 0,3 metros de profundidad. En la etiqueta antigua aparece como Anodonta cygnea variedad nobilis.

MNCN 15.07/3265: Sevilla?; Colección Ortiz de Zárate; SECO; 1 ej.

Obs.: En la etiqueta antigua aparece como Anodonta cygnea variedad ventricosa

MNCN 15.07/3270: Pantano de la Tranquera, Nuévalos; Zaragoza; 8/JUL/1995; J. De Andrés Cobeta; SECO; 1 ej.

MNCN 15.07/3274: Río Ebro, Cenicero; La Rioja; Colección Ortiz de Zárate; SECO; 1 ej.

Obs.: En la etiqueta antigua aparece como "Anodonta cignea" variedad mollis Drouët.

MNCN 15.07/3285: Río Ebro, Logroño; La Rioja; Colección Ortiz de Zárate; V. Lorza; SECO; 1 ej.

Obs : En la etiqueta antigua aparece como "Anodonta cignea".

MNCN 15.07/3286: Albufera de Valencia; Valencia; Colección Ortiz de Zárate; Siro de Fez; SECO; 2 ej. Obs.: En la etiqueta antigua Anodonta cygnea variedad anatina.

MNCN 15.07/3289: Albufera de Valencia; Valencia; Colección Ortiz de Zárate; Siro de Fez; SECO; 1 ej. 


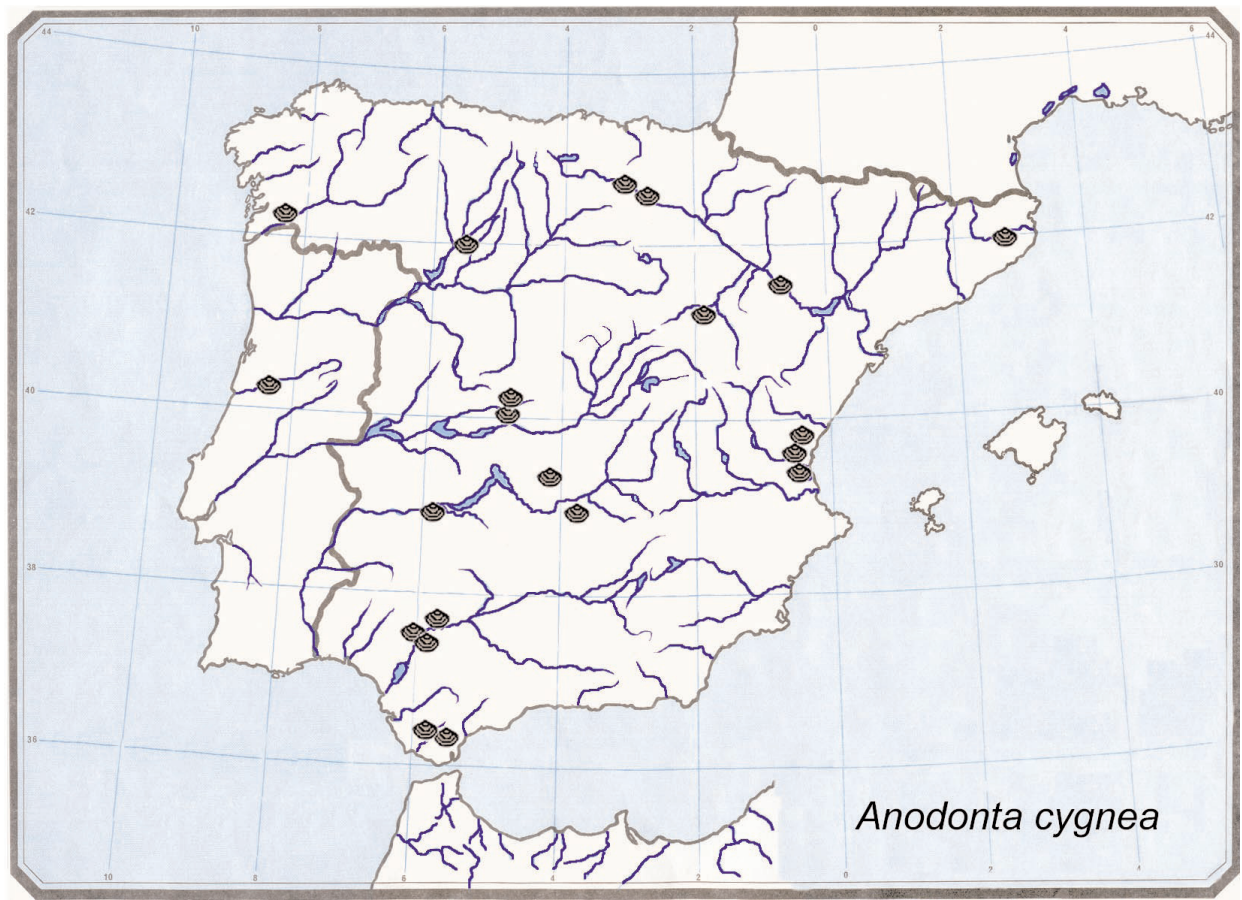

Fig. 6.- Distribución del material contenido en las Colecciones del MNCN de Anodonta cygnea (Linnaeus, 1758).

Fig. 6.- Distribution of the Anodonta cygnea (Linnaeus, 1758) material from the Spanish MNCN Collections.

Obs En la etiqueta antigua aparece como Anodonta cygnea var submacilenta Servain.

MNCN 15.07/5217: Embalse de Gasset; Ciudad Real; 11/NOV/1989; R. Araujo; SECO; 2 ej.

MNCN 15.07/5222: Río Guadamez, Vega de los Maderos, Don Benito; Badajoz; Verano/1941; SECO; 1 ej.

MNCN 15.07/5223: Río Alberche, Cazalegas; Toledo; Colección Leo Imperatori; SECO; 1 ej.

MNCN 15.07/5228; Albufera de Valencia; Valencia; Colección Azpeitia 1170; SECO; 1 ej.

Obs.: En la etiqueta antigua aparece como Anodonta valentina; Drouët, 1893. Material estudiado en Azpeitia (1933: 542-543).

MNCN 15.07/5229: Albufera de Valencia; Valencia; SECO; 1 ej. Obs.: El ejemplar lleva el número 4084 escrito en las dos valvas. En la etiqueta antigua aparece como Anodonta bicolor Drouët.

MNCN 15.07/5230: San Roque; Cádiz; Colección Cobos; AGO/1965; L. Ramírez García; SECO; 4 ej.

Obs.: Lote con dos etiquetas antiguas, en una de ellas pone que dos ejemplares proceden del arroyo de la Mujer, San Roque. En las etiquetas antiguas aparece como "Anodonta cygnea anatinia".

MNCN 15.07/5231: El Saler, canal de la Albufera de Valencia Valencia; Colección Cobos; 26/JUL/1968; L. Gasull; SECO; 2 ej.
MNCN 15.07/5250: Arroyo de la Mujer, San Roque; Cádiz; Colección Cobos; AGO/1965; L. Ramírez García; SECO; 1 ej.

Obs.: En la etiqueta antigua aparece como Anodonta cygnea anatina $(\mathrm{L}$.$) .$

MNCN 15.07/5286: Río Ebro, Villafranca de Ebro; Zaragoza; Colección Cobos; FEB/1967; SECO; 2 ej.

MNCN 15.07/5287: Río Cea, Valdescorriel: Zamora; Colección Cobos; 30/AGO/1978; L. Romero; 3 ej.

MNCN 15.07/5288: Charcas de verano; arroyo de la Mujer, San Roque; Cádiz; Colección Cobos; AGO/1978; L. Ramírez García; 1 ej.

MNCN 15.07/5289: El Palmar; Albufera de Valencia; Valencia; Colección Cobos; 15/AGO/1969; Gasull; 1 ej.

MNCN 15.05/5290: Canal del Saler, Albufera de Valencia, Valencia; Colección Cobos; Gasull; 1 ej.

MNCN 15.07/5291: San Roque; Cádiz; Colección Cobos; 24 ej.

\section{AGRADECIMIENTOS}

Queremos agradecer a la Dra. Vidal-Abarca y al Dr. Nagel la revisión crítica del manuscrito y al Dr. Márquez la revisión del resumen en inglés. 


\section{Referencias}

AzPeitia, F., 1933. Conchas bivalvas de agua dulce de España y Portugal. Memorias del Instituto Geológico y Minero de España.Tomos I y II. 763 pp + XXXVI lam.

Bogan, A.E., 1993. Freshwater bivalve extinctions (Mollusca: Unionida): A search for causes. American Zoologist, 33: 599-609.

HAAS, F., 1917. Estudio para una monografía de las náyades de la península Ibérica. Publicacions de la Junta de Ciencies Naturals de Barcelona; anuari II. Barcelona. 131-139.

HAAS, F., 1969. Superfamilia Unionacea. Das Tierreich. Vol. 88. Walter de Gruyter \& Co. (Ed.). Berlin. 663 pp.

Kaufman, L., 1992. Catastrophic change in species-rich freshwater ecosystems. Bioscience, 42(11): 846-858.

LOCARD, A., 1899. Conchyliologie portugaise: Coquilles terrestres, des eaux douces et saumâtres. Archives $d u$ Musée d'Histoire Naturelle de Lyon, 7: 1-303.

Morelet, A., 1845. Description des Mollusques terrestres et fluviatiles du Portugal. J. B. Bailliere. París. 1 vol. $8^{\circ}, 116$ pp. + 14 lám.
Nagel, K.O., Badino, G. \& Alessandria, B., 1996. Population Genetics of European Anodontinae (Bivalvia: Unionidae). Journal of Molluscan. Studies, 62:343-357.

Nagel, K.O., Badino, G. \& Celebrano, G., 1998. Systematics of European naiades (Bivalvia: Margaritiferidae and Unionidae): a review and some new aspects. Malacological Review, Supplement 7, Bivalvia I: 83-104.

Simpson, C.T., 1900. Synopsis of the Naiades or Pearly Fresh-water Musels. Charles Torrey \& Washington Government Printing Office. Washington. 272 pp.
Recibido, el 15-V-2001 Aceptado, el 13-VII-2001 Publicado, el 20-VIII-2001 